\title{
Flow of buoyant granular materials along a free surface
}

\author{
Zhong Zheng $g^{1,2,3} \dagger$, Herbert E. Huppert $\mathbf{t}^{1,4,5}$, \\ Nathalie M. Vriend ${ }^{1}$, Jerome A. Neufeld $d^{1,2,3}$, \\ AND P. F. Linde $\mathbf{n}^{1}$ \\ ${ }^{1}$ Department of Applied Mathematics and Theoretical Physics, \\ University of Cambridge, Cambridge CB3 0WA, UK \\ ${ }^{2}$ BP Institute, University of Cambridge, Cambridge, CB3 0EZ, UK \\ ${ }^{3}$ Department of Earth Sciences, University of Cambridge, Cambridge CB3 0EZ, UK \\ ${ }^{4}$ Faculty of Science, University of Bristol, Bristol BS2 6BB, UK \\ ${ }^{5}$ School of Mathematics and Statistics, University of New South Wales, NSW 2052, AU
}

(Received January 10, 2018; revised ?; accepted ?. - To be entered by editorial office)

We study experimentally the flow of light granular material along the free surface of a liquid of greater density. Despite a rich set of related geophysical and environmental phenomena, such as the spreading of calved ice, volcanic ash, debris and industrial wastes, there are few previous studies on this topic. We conduct a series of lock-release experiments of buoyant spherical beads into a rectangular tank initially filled with either fresh or salt water, and record the time evolution of the interface shape and the front location of the current of beads. We find that following the release of the lock the front location obeys a power-law behaviour during an intermediate time period before the nose of beads reaches a maximum runout distance within a finite time. We investigate the dependence of the scaling exponent and runout distance on the total amount of beads, the initial lock length, and the properties of the liquid that fills the tank in the experiments. Scaling arguments are provided to collapse the experimental data into universal curves, which can be used to describe the front dynamics of buoyant granular flows with different size and buoyancy effects and initial lock aspect ratios.

Key words: granular media, geophysical and geological flows, gravity currents

\section{Introduction}

Inspired by aspects of the runout of granular materials such as ice from glacier calving, debris from land slides, dredged materials, industrial wastes, volcanic ash and snowladen air and similar phenomena, we study the flow of granular material along the upper surface of a liquid. Key open questions include the generation of tsunami waves once the granular materials hit the surface of an ocean, lake or river, and how the granular materials spread after the initial impact. Here we investigate the case of the flow of buoyant granular material in a channel containing a liquid of a greater density, so that the granular material floats near the air-liquid interface. Such flows have only been considered for simple fluids, for example, in the viscous limit in the context of oil spreading in the sea, and ice shelf and grounding lines dynamics (see, e.g., Hoult 1972; Lister \& Kerr 1989; Pegler \& Worster 2013). Similar phenomena also appear in freshwater rivers flowing into

$\dagger$ Email address for correspondence: zzheng@alumni.princeton.edu 
salt-water oceans and intrusive gravity currents entering stratified environments (see, e.g., Holyer \& Huppert 1980; de Rooij et al. 1999; Maurer et al. 2010; Carazzo \& Jellinek 2012).

Related previous studies on this topic also include the collapse of heavy beads along an inclined substrate into a shallow layer of water, inspired by the landslide process (see, e.g., Viroulet et al. 2014; Zitti et al. 2016). The major focus of Viroulet et al. (2014) and Zitti et al. (2016) is on the generation of tsunami waves caused by the impact of the granular material on the shallow water. The spreading of heavier granular materials into a water-filled channel has also been investigated through a series of experiments with different initial conditions and granular materials (Hallworth \& Huppert 1998; Pailha et al. 2008; Rondon et al. 2011) and through theoretical models (see, e.g., Pailha \& Pouliquen 2009) and numerical simulations (Topin et al. 2012). Similar to the situation of dry granular spreading, investigated experimentally (Lube et al. 2004, 2005; Balmforth \& Kerswell 2005; Thompson \& Huppert 2007) and theoretically (Savage \& Hutter 1989; Pouliquen \& Hutter 2002; Staron \& Hinch 2005; Larrieu et al. 2006; Staron \& Hinch 2007), in experiments of granular collapse within a liquid of lower density, a final profile shape was observed at late times with a finite runout distance of the granular material. However, when the viscosity of the ambient liquid becomes important, the dynamics of granular collapse is modified from the case of dry granular collapse (du Pont et al. 2003; Pailha \& Pouliquen 2009; Topin et al. 2012).

Another related topic concerns particle-laden gravity currents driven by the density difference between the particles and the ambient liquid (see, e.g., Bonnecaze et al. 1993, 1995). In these flows there is also a finite runout distance, because the heavy particles settle out from the current so that the bulk density difference eventually becomes zero and the current stops. The speed of particle sedimentation is typically determined by a balance between the gravitational force and viscous drag (Bonnecaze et al. 1993). We also note that the particle concentrations in these particle-laden flows are typically much lower than the particle concentrations in the granular collapse experiments of Hallworth \& Huppert (1998) and the granular intrusion experiments described in the current work.

In this study we focus on the temporal evolution of relatively light beads spreading from a lock into a relatively denser ambient liquid. We describe a series of experiments of interfacial granular intrusions into a water tank in $\S 2$. We interpret the major observations on the time-dependent profile shape, front location and runout distance in $\S 3$. We also provide scaling arguments to rescale and collapse the experimental data into universal curves, which capture the major physics during the spreading process. We summarize the major findings in $\S 4$, and comment on open questions and future directions and the major differences between the interfacial granular intrusion and three other related problems: the intrusive gravity current, the particle-laden gravity current and the dry granular collapse problems.

\section{Experimental Design}

We examine the spreading of a constant volume of buoyant particles, floating on a liquid of relatively greater density. The experiments were conducted in a rectangular tank of length $l_{t}=2.09 \pm 0.01 \mathrm{~m}$, width $w_{t}=0.15 \pm 0.01 \mathrm{~m}$ and depth $d_{t}=0.57 \pm 0.01 \mathrm{~m}$, as sketched in figure 1. Before each experiment, the tank was filled with either fresh water, density $\rho_{w}=998 \mathrm{~kg} \cdot \mathrm{m}^{-3}$, or saturated sodium chloride solution, density $\rho_{s w}=1,180$ $\mathrm{kg} \cdot \mathrm{m}^{-3}$. The water depth $d$ was set to $d=15$ or $20 \mathrm{~cm}$. A lock gate of small thickness $(2.5$ $\mathrm{mm}$ ) was placed at a location $x_{i}$ (i.e., the lock length), measured from one end of the tank (i.e., from the origin at $x=0$ ), as shown in figure $1 a$. The gap between the lock gate and 

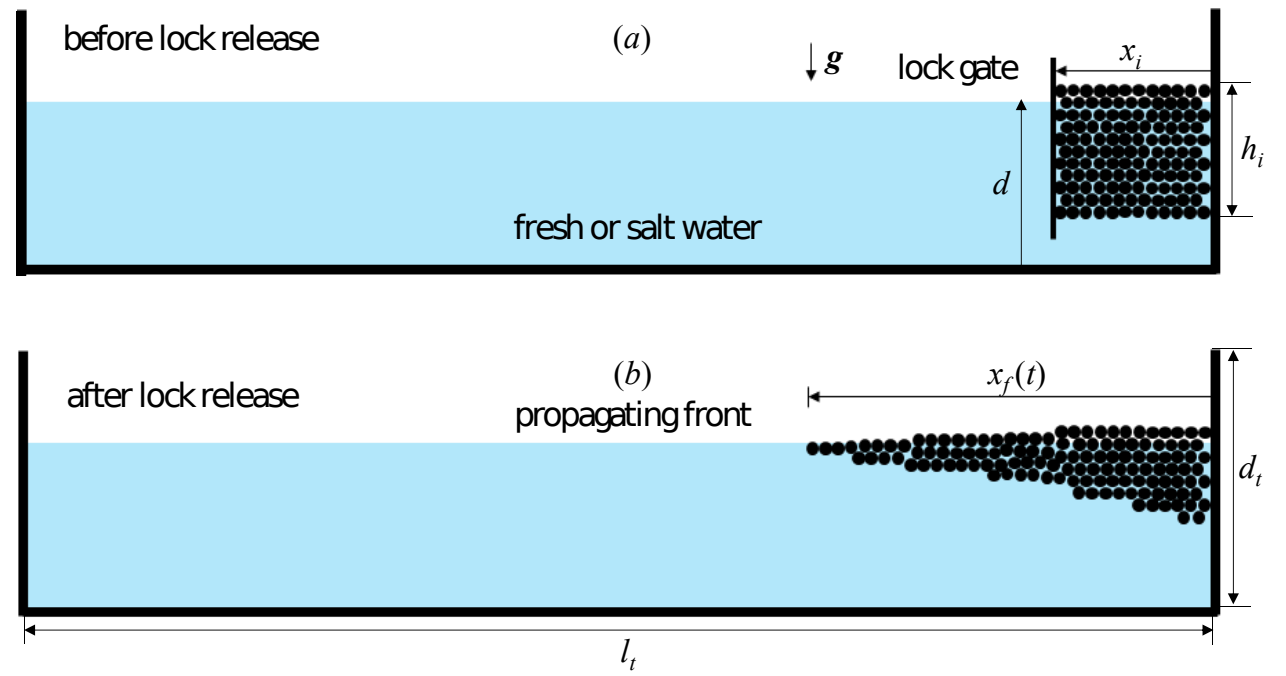

Figure 1. Sketch of the experimental setup $(a)$ before and $(b)$ after release of the lock gate. The lock length is denoted by $x_{i}$, while the front location is denoted by $x_{f}(t)$.

the origin was filled with a mass $m$ of spherical polypropylene beads (McMaster Carr, $1974 \mathrm{~K} 2$ ) of radius $r=1.6 \mathrm{~mm}$ and density $\rho=910 \mathrm{~kg} \cdot \mathrm{m}^{-3}$. The beads were carefully maintained in an initial rectangular shape before the removal of the lock gate (figure $1 a$ ).

To start the experiments, we quickly (within $0.1 \mathrm{~s}$ ) lifted the lock gate vertically and the beads spread horizontally along the surface of the liquid (figure 1b). A digital camera was used to take videos from either the side or above the tank. Digiflow software (Dalziel 2005) and MATLAB image processing toolbox were used to analyse videos and extract the interface shape between the beads and water and the location of the front of the current of beads. In order to explore buoyancy effects, we used either fresh water or saturated salt water as the ambient liquid in the tank. We also varied the depth of the liquid $d$ and the total amount of beads $m$. In addition, to explore the influence of the initial aspect ratio of the beads, we kept the mass of beads $m$ constant but varied the initial lock length $x_{i}$. The experimental parameters are given in table 3 and the results are summarized in table 4 in Appendix A. The error estimates on the measured parameters in table 3 and thereafter come from the standard deviation of repeated experiments.

\section{Experimental Results}

\subsection{Interface shape}

Side and top views of two representative experiments are shown in figures 2 and 3, respectively. In these two experiments with identical parameters, the tank was filled with saturated salt water of depth $d=15 \pm 0.1 \mathrm{~cm}$, the lock length was $x_{i}=10 \mathrm{~cm}$, and the total mass of the beads was $m=765 \pm 1 \mathrm{~g}$.

As can be seen from figure 2, the current of beads (soaked in water) propagates along the free surface and culminates in a very shallow front which is one or two beads in thickness. Eventually the front of the beads reaches a maximum location, all motion stops and the interface shape stays unchanged at later times. From the top view, shown in figure 3, we observe that the propagating front of the light beads remains almost planar across the width of the tank. The top-view pictures also indicate that the front can become irregular towards the end of the experiments when the spreading rate is very 

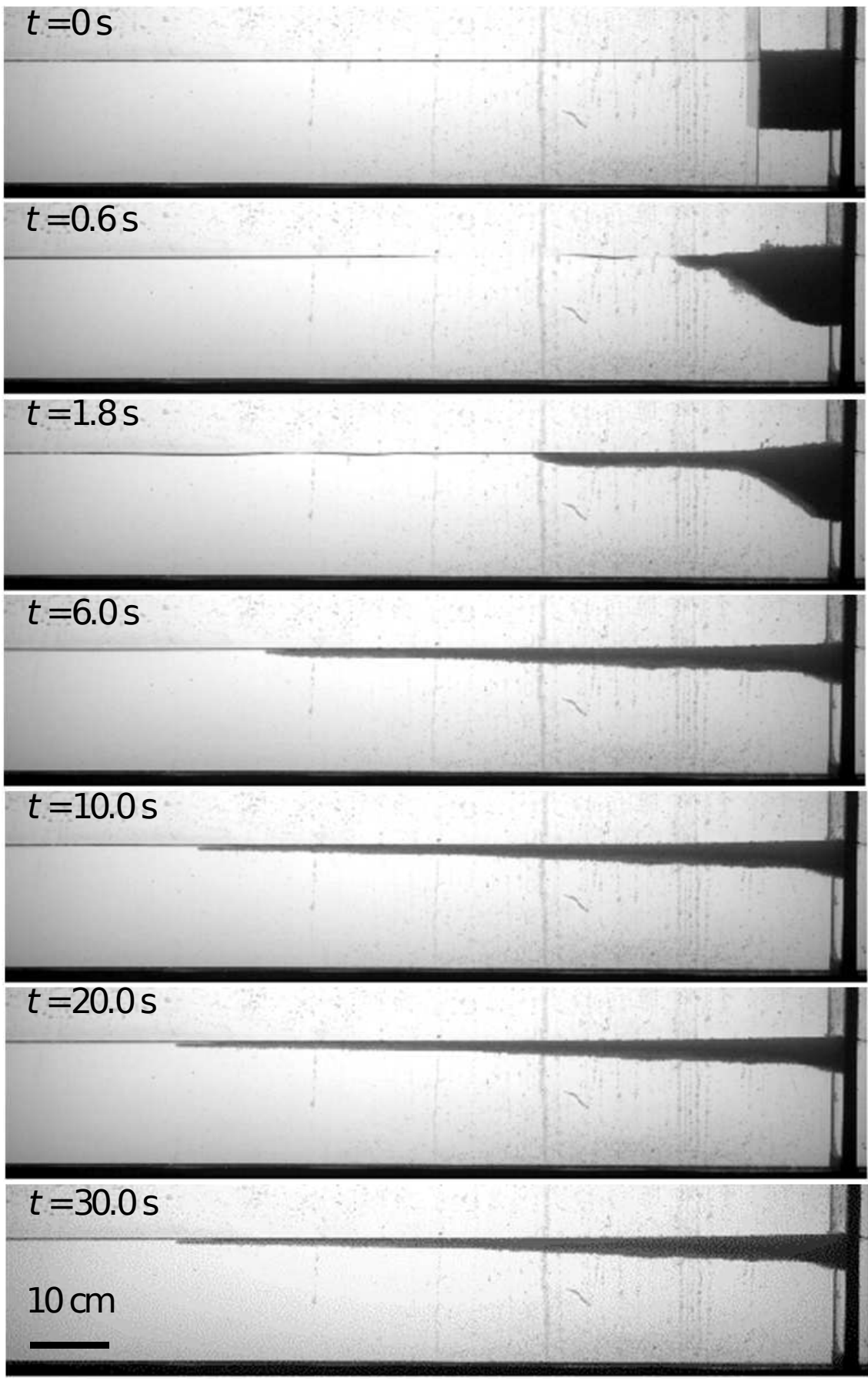

FiguRE 2. Side views of the spreading beads in a representative experiment (Expt $2 \mathrm{a}$ in table 3 ). The pictures were taken at $t=\{0,0.6,1.8,6.0,10,20,30\} \mathrm{s}$. The tank was filled with saturated salt water of depth $d=15 \pm 0.1 \mathrm{~cm}, m=765 \pm 1 \mathrm{~g}$, and $x_{i}=10 \mathrm{~cm}$. The pictures show the initial spreading with time followed by the front coming to a halt. 

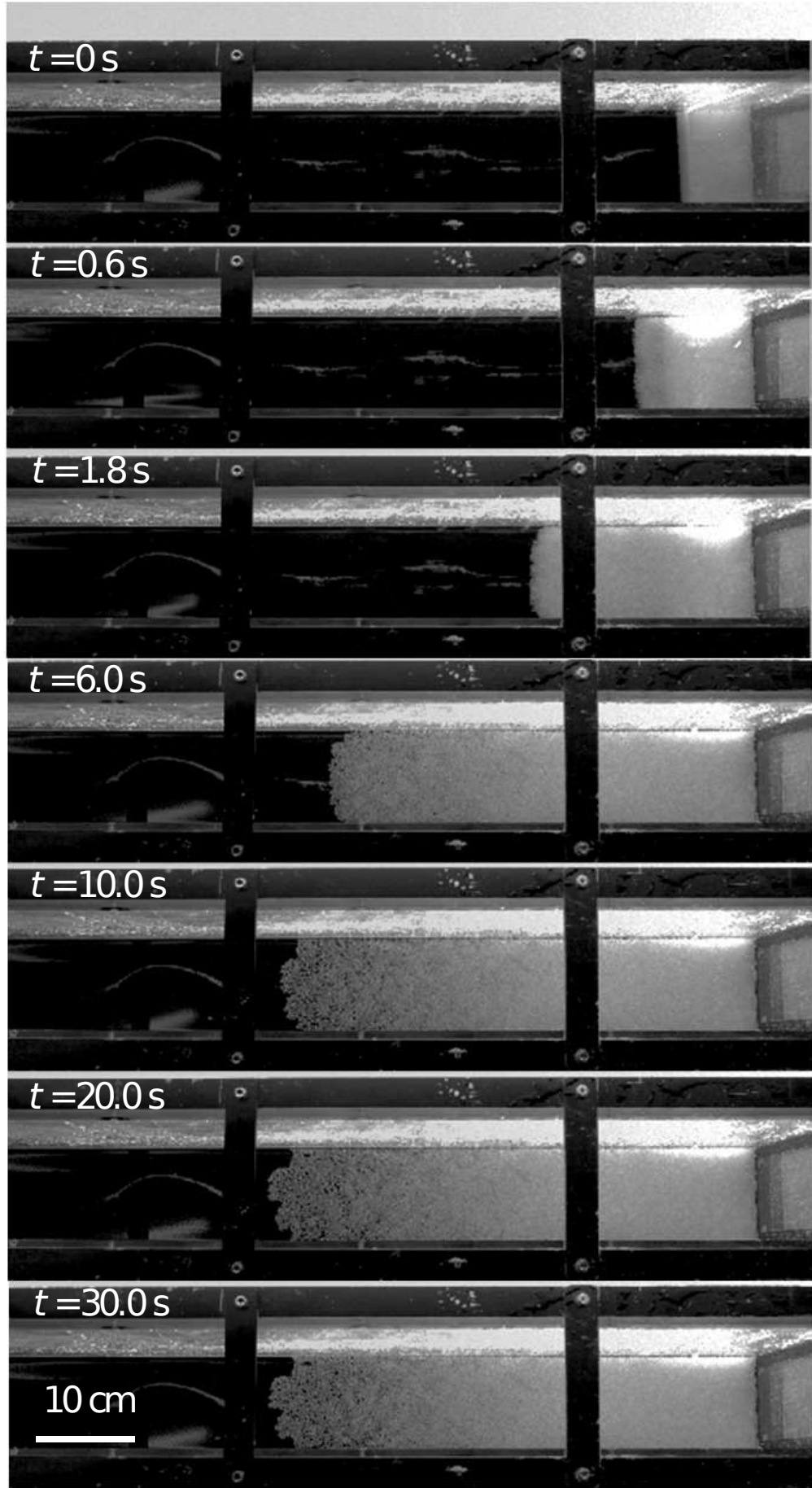

FIGURE 3. Top view of the spreading beads in a representative experiment. The propagating front remains almost uniform across the width of the tank until the current is close to its final length. The pictures are taken at $t=\{0,0.6,1.8,6.0,10,20,30\} \mathrm{s}$. The experimental parameters are the same as the experiment in figure 2 . 


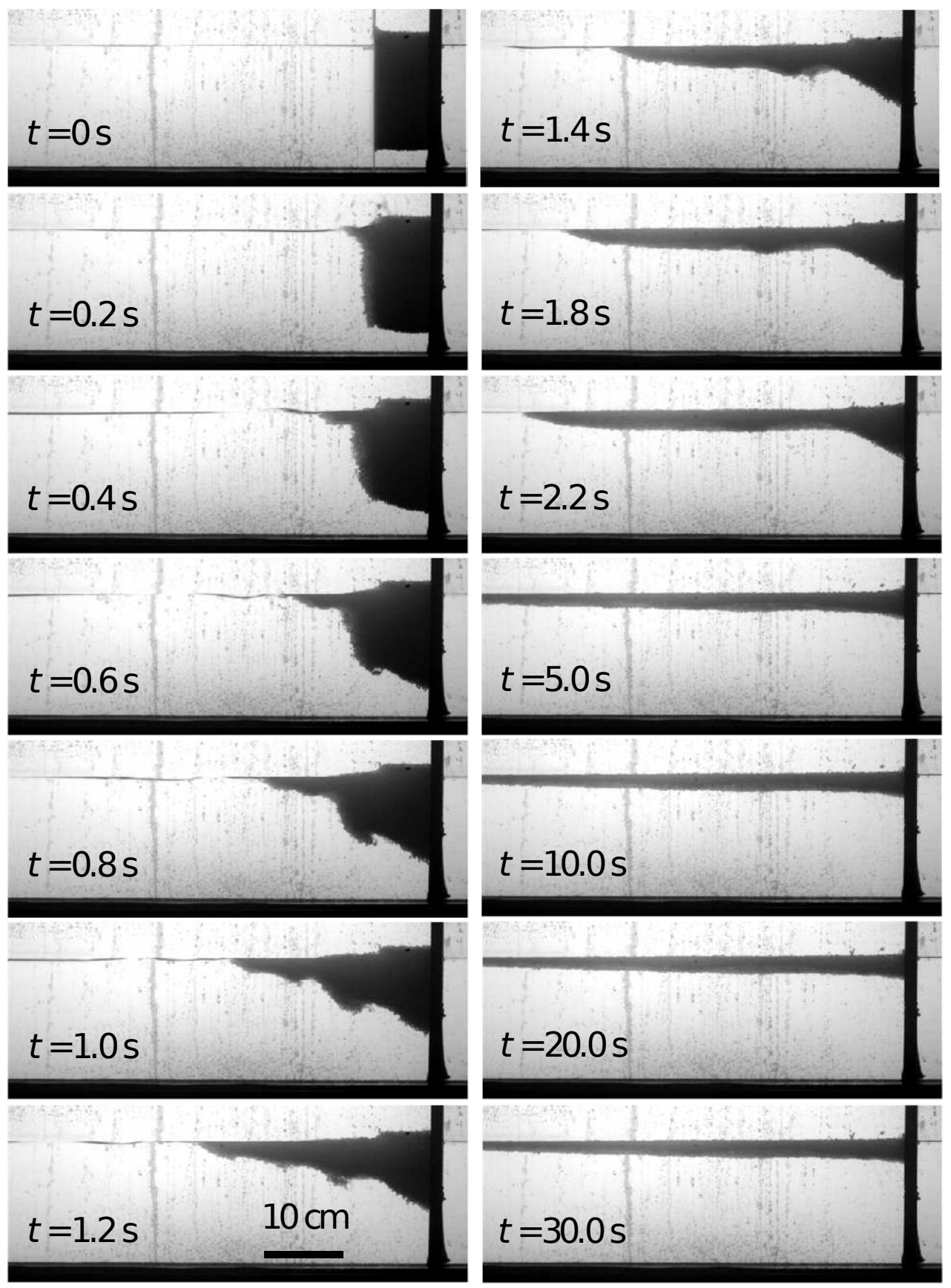

FiguRE 4. For small initial lock lengths (e.g., $x_{i} \leqslant 10 \mathrm{~cm}$ ), the interface shape exhibits an almost constant slope near the origin between $t=0.6 \mathrm{~s}$ and $t=2.2 \mathrm{~s}$ before decreasing to a smaller angle at the end. Shear-driven instabilities also appear at the interface at the beginning of the experiments. This experiment (not included in table 3) was conducted using saturated salt water of depth $d=15 \pm 0.1 \mathrm{~cm}, m=765 \pm 1 \mathrm{~g}$, and $x_{i}=5 \mathrm{~cm}$. 


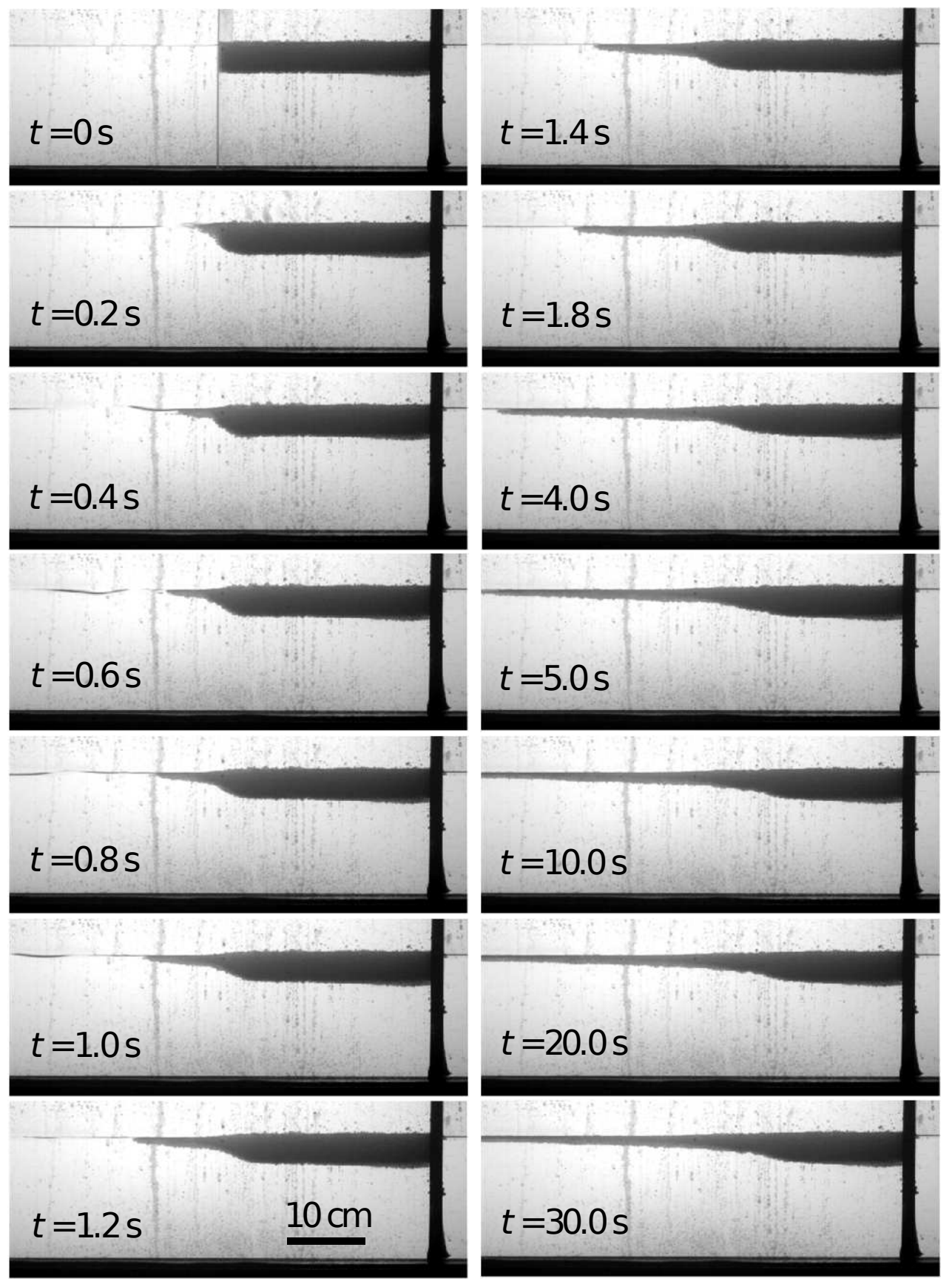

Figure 5. For large initial lock lengths (e.g., $x_{i} \geqslant 20 \mathrm{~cm}$ ), there exists a "frozen" region near the origin where the interface shape stays unchanged. This experiment (not included in table 3) was conducted using saturated salt water of depth $d=15 \pm 0.1 \mathrm{~cm}, m=765 \pm 1 \mathrm{~g}$, and $x_{i}=25$ $\mathrm{cm}$. 

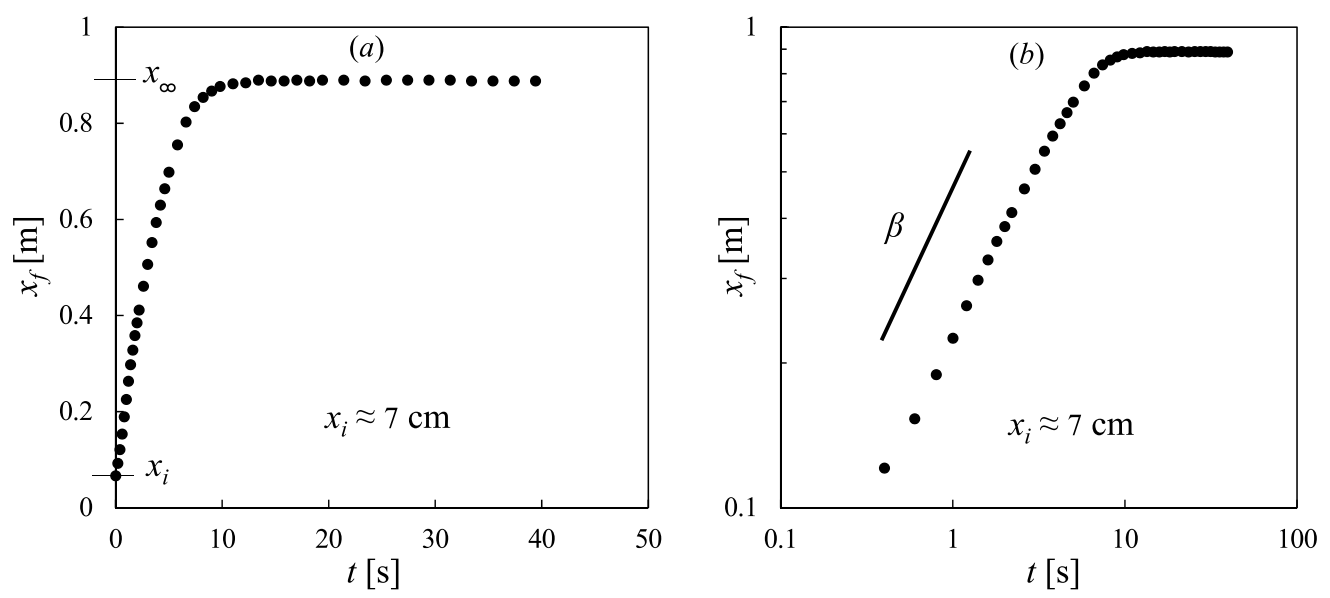

FIGURE 6 . Time evolution of the propagating front in a representative experiment (Expt $1 a$ in table 3). (a) The flow is arrested at long times and a runout distance $x_{\infty}$ can be defined. (b) A constant slope $\beta$ is observed during an intermediate period in the log-log plot, which suggests the scaling of $x_{f}(t) \propto t^{\beta}$, with $\beta \approx 0.6$ in this example.

slow, and the effects of surface tension, ambient viscosity and sidewall friction may have become significant. Nevertheless, in general, the roughness of the front is small compared with the length of the current, so we neglect the variations of the front across the channel width, and define the front location $x_{f}(t)$ based on the side view pictures, shown in figure 2 .

In addition, from the top view (figure 3), we observe that the propagating front of the light beads remains almost planar across the width of the tank. The front can become irregular near the end of the experiments, and the effects of surface tension may have become significant. Nevertheless, we neglect the variations of the propagating front across the channel width, and define the front location $x_{f}(t)$ based on the side view pictures (figure 2) and provide a detailed quantitative description for the time evolution of the front location $x_{f}(t)$ in $\S 3.2$.

When the lock length is small $\left(x_{i} \leqslant 10 \mathrm{~cm}\right)$ and hence, the initial depth is large, near the origin the interface shape contains a region with an almost constant slope (figure 4). This is different from the observations in the fixed-volume lock-release experiments of Newtonian liquids spreading along a free surface in which the slope remains zero at the origin (Lister \& Kerr 1989). We also note, for small lock lengths, that a shear-driven instability typically appears along the bottom of the current at the beginning of the experiments (figure 4).

When the lock length is large $\left(x_{i} \geqslant 20 \mathrm{~cm}\right)$ and the initial depth is small, the interface shape contains a "frozen region" near the origin, with an interface shape that remains almost unchanged from its initial state during the entire flow (figure 5). Similar phenomena have also been observed in the fixed-volume lock-release experiments of dry granular materials along a horizontal substrate and the deposits look like a "Mexican hat" (Lube et al. 2004; Lajeunesse et al. 2004). We also note that a shear-driven instability typically appears at the interface of the water and beads at the beginning of the experiments (figure 4), when the initial lock length is small. 

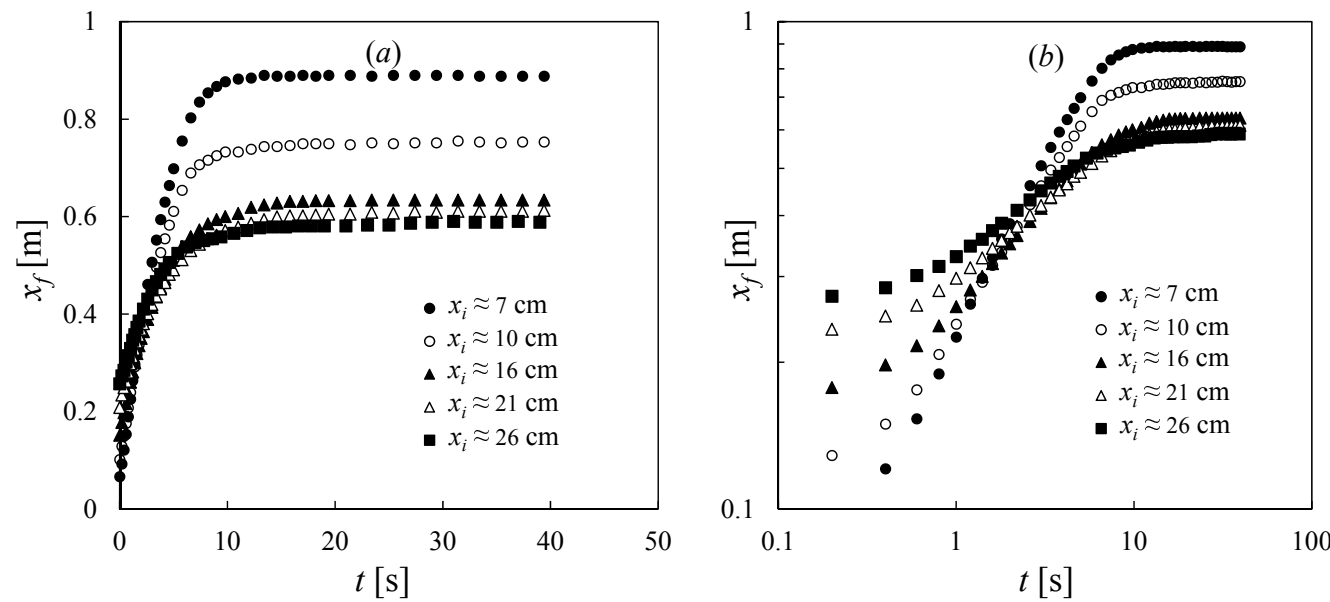

FiguRE 7 . Time evolution of the propagating front $x_{f}(t)$ in different experiments, which demonstrates the influence of the lock length. In these experiments, the tank was filled with saturated salt water of depth $d=15 \pm 0.1 \mathrm{~cm}, m=765 \pm 1 \mathrm{~g}$, and $x_{i} \approx\{7,10,16,21,26\} \mathrm{cm}$. The influence of the lock length $x_{i}$ on the runout distance $x_{\infty}$ in the arrested state, and scaling exponent $\beta$ in the intermediate period can be observed.
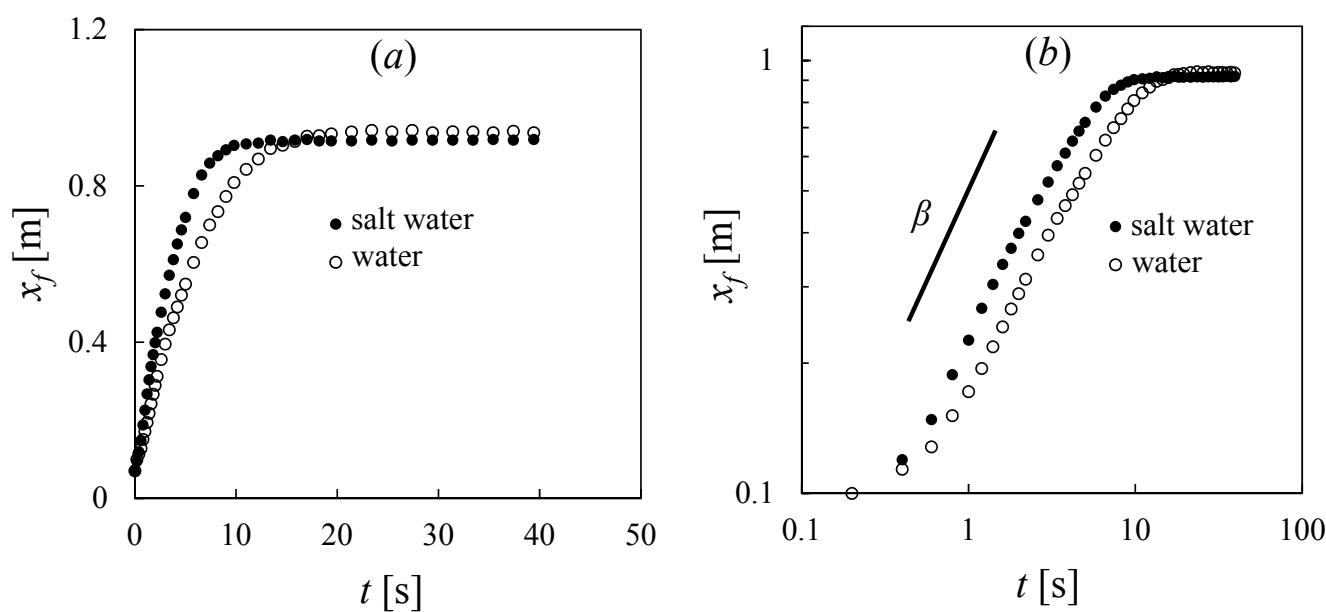

FiguRE 8. Time evolution of the propagating front $x_{f}(t)$ in different experiments, indicating the influence of the density of the ambient liquid. The tank was filled with either water or salt water of depth $d=15 \pm 0.1 \mathrm{~cm}, m=765 \pm 1 \mathrm{~g}$, and $x_{i}=5 \mathrm{~cm}$ in both experiments. During the intermediate period, when $x_{f}(t)=\alpha t^{\beta}$, the scaling exponent $\beta$ in both experiments was found to be almost the same, while the prefactor $\alpha$ differed. The runout distance $x_{\infty}$ in the two experiments were also slightly different.

\subsection{Propagating front}

The time evolution of the location of the propagating front $x_{f}(t)$ for a representative experiment, shown in (figure 6). After an initial acceleration from rest, we observed a constant slope $\beta$ in the log-log plot during an intermediate time period ( $1 \mathrm{~s} \leqslant t \leqslant 5 \mathrm{~s}$ ), as shown in figure $6 b$, which indicates a scaling behaviour for the front location $x_{f}(t) \propto t^{\beta}$. Then, the spreading slowed down, and the front location approached a final value $x_{\infty}$, defined as the run-out distance, as shown in figure $6 a$, which corresponds to the final arrested state. 

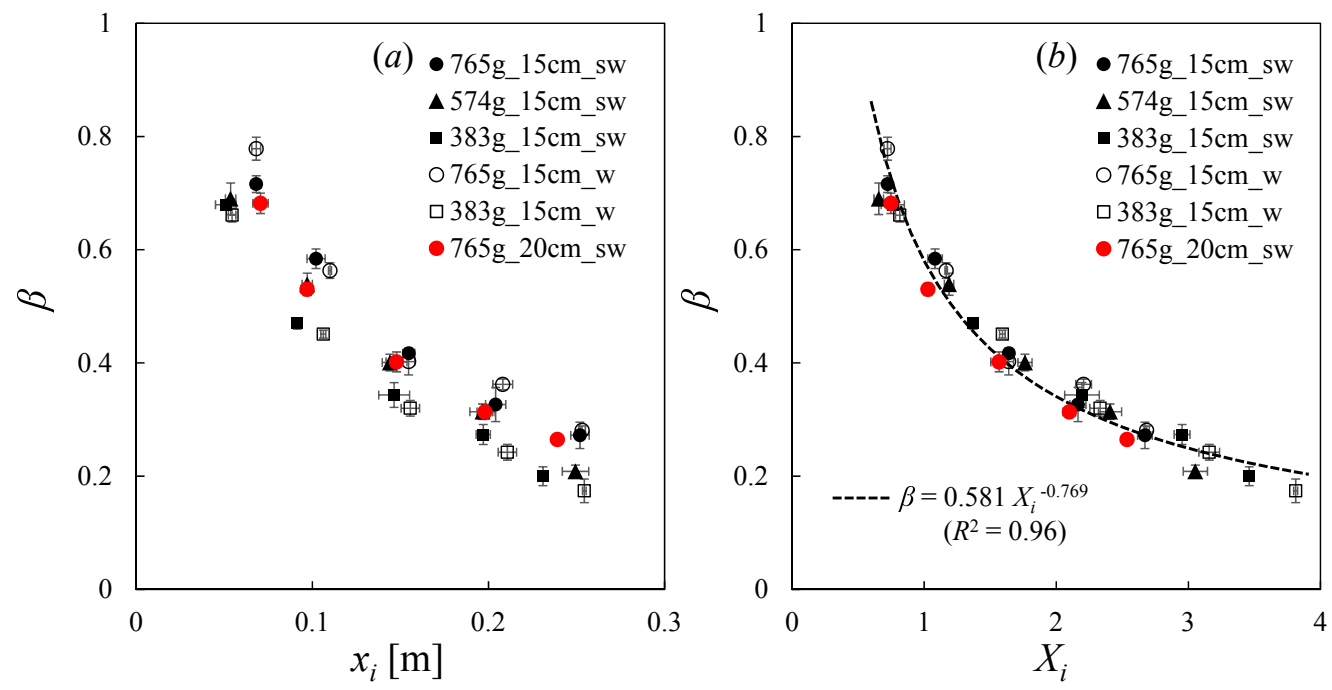

FIGURE 9. The scaling exponent $\beta$ in $x_{f}(t)=\alpha t^{\beta}$ from different experiments during an intermediate period after the removal of the lock gate: $(a)$ raw data and $(b)$ rescaled data. The rescaled data collapse to a universal curve and the dashed curve represents a best power-law fit. The definition of the scaling exponent $\beta$ is demonstrated in figure $6 b$. The dimensionless lock length is $X_{i} \equiv x_{i} / l_{c}$ with the characteristic length scale $l_{c}$ defined in (3.1), which accounts for the total mass of the beads $m$.

The influence of the lock length $x_{i}$ on the front location $x_{f}(t)$ is shown in figure 7 . The data show that for a fixed amount of beads, an increase in the initial local length $x_{i}$ corresponds to a decrease in both the scaling exponent $\beta$ during the intermediate period and the runout distance $x_{\infty}$ at the final arrested state. We note that the decrease in the runout distance $x_{\infty}$ appears progressively weaker, and $x_{\infty}$ eventually approaches a constant, as we further increase the lock length $x_{i}$ beyond a critical value. We also note that the capillary effects appear to become increasingly significant when we continue to increase the lock length $x_{i}$, as suggested by an irregular propagating front (which contains only one layer of beads) from the top view.

The influence of the fluid density on the front location $x_{f}(t)$ is shown in figure 8. During the intermediate period, when the front location obeys $x_{f}(t)=\alpha t^{\beta}$, the scaling exponent $\beta$ in both experiments appears to be the same, while the prefactor $\alpha$ differs, and a stronger buoyancy effect corresponds to a greater prefactor $\alpha$. In addition, the runout distances $x_{\infty}$ in the two experiments are slightly different, which is not statistically significant.

\subsubsection{Scaling exponent $\beta$}

The scaling exponent $\beta$ is plotted against the lock length in figure $9 a$. Within the parameter range we considered, the experiments show a negligible influence of water depth $d$, and buoyancy $\Delta \rho g$. On the other hand, for a fixed amount of beads $m$, the scaling exponent $\beta$ decreases as the lock length $x_{i}$ increases, as mentioned before. In addition, the total amount of beads $m$ has a systematic influence on the scaling exponent $\beta$ : an increase in $m$ corresponds to an increase in $\beta$, and the same dependence holds for experiments in both water and salt water.

In order to address the effect of initial mass, we define a dimensionless lock length as 

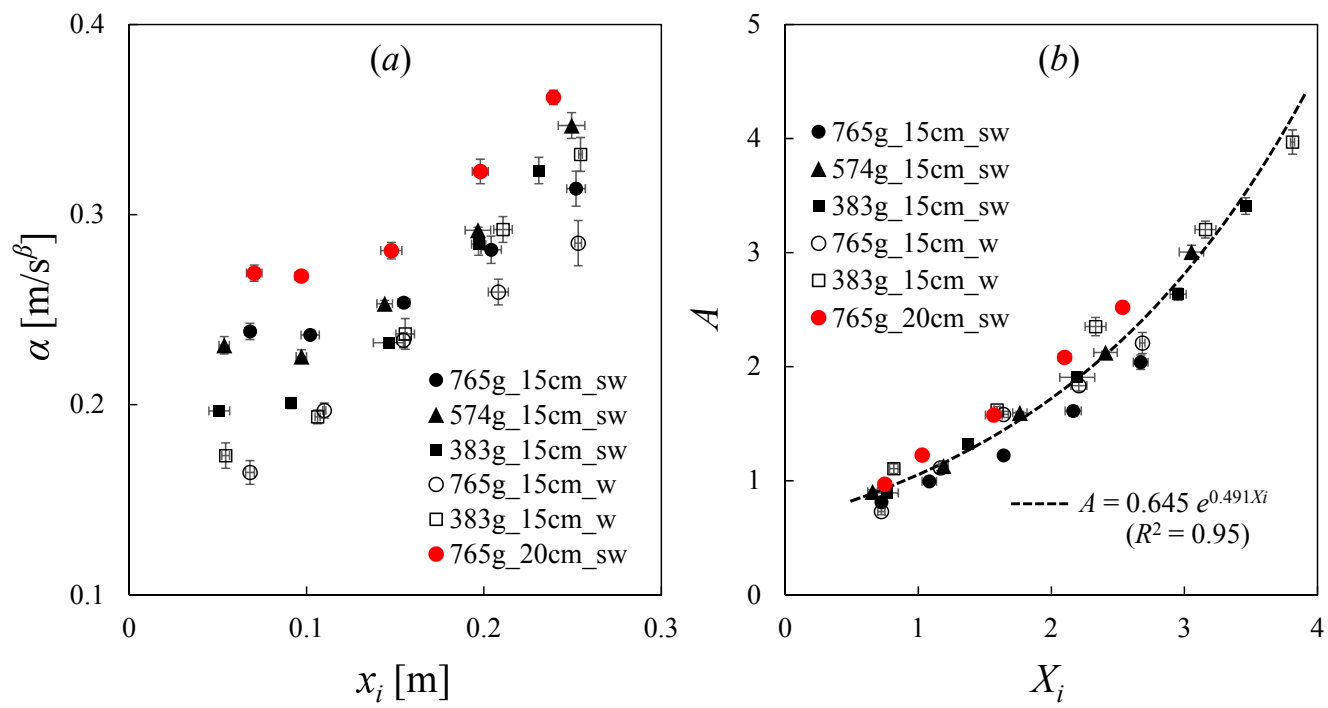

FiguRE 10. The prefactor $\alpha$ in $x_{f}(t)=\alpha t^{\beta}$ from different experiments during an intermediate period after the removal of the lock gate: $(a)$ raw data and $(b)$ rescaled data. The dashed curve is a best exponential fit. The dimensionless lock length is $X_{i} \equiv x_{i} / l_{c}$ with the characteristic length scale $l_{c}$ defined in (3.1), which accounts for the total mass of the beads $m$. The dimensionless prefactor $A \equiv \alpha / \alpha_{c}$ with the characteristic scale $\alpha_{c}$ defined in (3.3), includes the effects of both the total mass of beads $m$ and the buoyancy $\Delta \rho g$.

$X_{i} \equiv x_{i} / l_{c}$, based on a characteristic length scale $l_{c}$ defined by

$$
l_{c} \equiv\left[\frac{m}{\rho_{b} w_{t}(1-\phi)}\right]^{1 / 2}=\left(x_{i} h_{i}\right)^{1 / 2},
$$

where $\rho_{b}$ is the density of the beads, $h_{i}$ is the initial vertical extent of the beads in the lock and $\phi$ is the porosity of the packed beads (the volume fraction occupied by liquid). We assume that initially the beads are closely packed spheres of equal size and take $\phi=0.37$ (Acton et al. 2001).

From the definitions of $l_{c}$ and $X_{i}$, we obtain that $X_{i}=\left(x_{i} / h_{i}\right)^{1 / 2}$, which measures the inverse aspect ratio (height/length) of the initial arrangement of beads. The power law exponent $\beta$ is plotted versus $X_{i}$ in figure $9 b$. This produces a collapse of the data and shows that $\beta$ decreases as the lock aspect ratio decreases. A least squares best fit of $\beta$ as a power of $X_{i}$ gives

$$
\beta=0.581 X_{i}^{-0.769}, \quad \text { for } 0.6 \leqslant X_{i} \leqslant 3.8,
$$

with $R^{2}=0.96$, and is shown as the dashed curve in figure $9 b$. Other fits to the experimental data are provided in Appendix B.

\subsubsection{Prefactor $\alpha$}

The prefactor $\alpha$ is plotted against $x_{i}$ in figure $10 a$. The value of $\alpha$ increases with $x_{i}, m$ and $\Delta \rho g$. Clearly, there is a systematic dependence on the initial lock length $x_{i}$, the total mass of beads $m$, and the buoyancy $\Delta \rho g$. To address these effects, we rescale the lock length $x_{i}$ based on the length scale $l_{c}$, defined in (3.1). To find an appropriate characteristic scale for $\alpha$, we assume that it depends only on the length scale $l_{c}$ and the reduced gravity $g^{\prime} \equiv \Delta \rho g / \rho_{l}$, where $\rho_{l}$ is the density of the liquid in the tank and $\rho_{l}=\rho_{w}$ or $\rho_{s w}$, respectively. Dimensional analysis suggests that we can define a dimensionless 


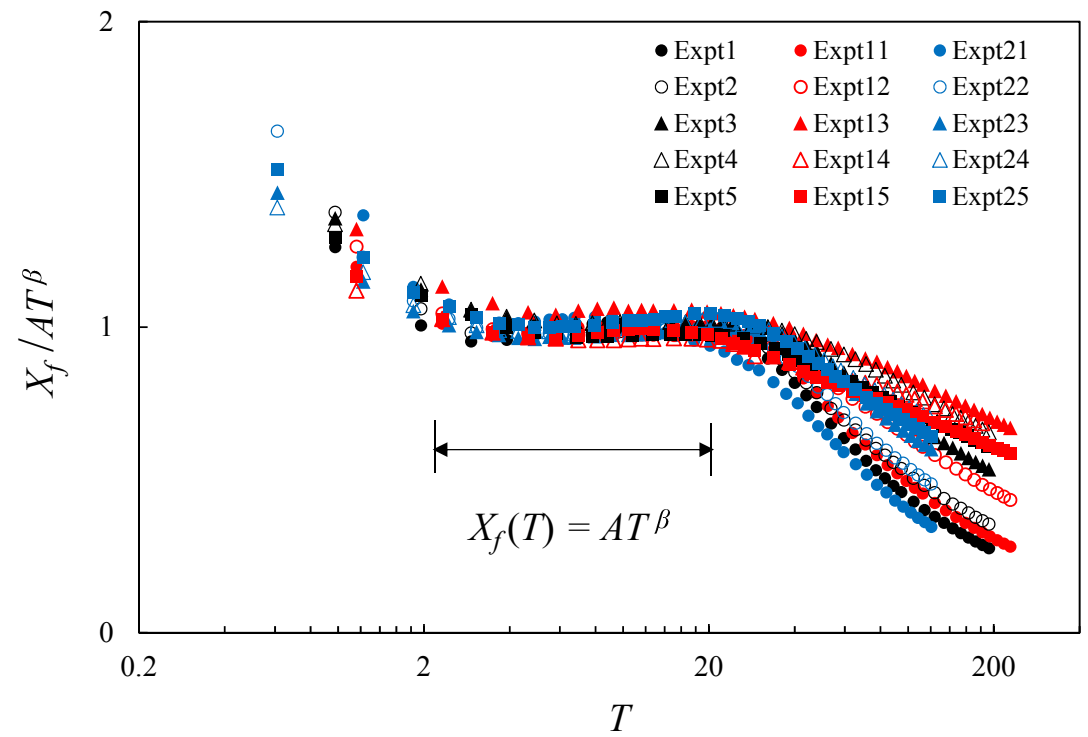

FiguRE 11. Measurements of the front location rescaled according to $X_{f}(T)=A T^{\beta}$. We observe good data collapse in an intermediate time period (approximately $2 \leqslant T \leqslant 20$ ). The approach toward and departure from the scaling behaviour are also demonstrated. The black $(m=765 \pm 1$ $\mathrm{g})$ and red $(m=383 \pm 1 \mathrm{~g})$ symbols indicate experiments in salt water and the blue $(m=765 \pm 1$ g) symbols represent experiments in fresh water. The detailed experimental parameters are listed in table 3 .

prefactor $A \equiv \alpha / \alpha_{c}$, where

$$
\alpha_{c} \equiv g^{\prime \frac{\beta}{2}} l_{c}^{1-\frac{\beta}{2}}=\left(\frac{\Delta \rho g}{\rho_{l}}\right)^{\frac{\beta}{2}}\left[\frac{m}{\rho w_{t}(1-\phi)}\right]^{\frac{1}{2}-\frac{\beta}{4}} .
$$

We plot the rescaled prefactor $A$ as a function of the rescaled lock length $X_{i}$ in figure $10 b$. This scaling collapses the data and shows that $\alpha$ decreases as the aspect ratio of the initial bead configuration increases (figure 10b). A best exponential fit for the data is

$$
A=0.645 e^{0.491 X_{i}}, \quad \text { for } 0.6 \leqslant X_{i} \leqslant 3.8,
$$

with $R^{2}=0.95$, and is shown as the dashed curve in figure $10 \mathrm{~b}$. Other best-fits using different functional forms of $A$ vs $X_{i}$ are shown in Appendix B.

\subsubsection{Propagation law}

The length scale $l_{c}$ and reduced gravity $g^{\prime}$ provide a characteristic time scale

$$
t_{c} \equiv\left(\frac{l_{c}}{g^{\prime}}\right)^{\frac{1}{2}}=\left(\frac{\Delta \rho g}{\rho_{l}}\right)^{-\frac{1}{2}}\left[\frac{m}{\rho w_{t}(1-\phi)}\right]^{\frac{1}{2}} .
$$

Defining a dimensionless time $T \equiv t / t_{c}$ and a dimensionless front location $X_{f}(T) \equiv x_{f} / l_{c}$, the time evolution of the front location $x_{f}(t)=\alpha t^{\beta}$ can be rewritten in a dimensionless form

$$
X_{f}(T)=A T^{\beta} .
$$

The front locations rescaled according to (3.6) are shown in figure 11. As expected the data collapse during the period of power law spreading $(2 \leqslant T \leqslant 20)$. The approach toward and departure from the scaling behaviour are also demonstrated in figure 11 . 

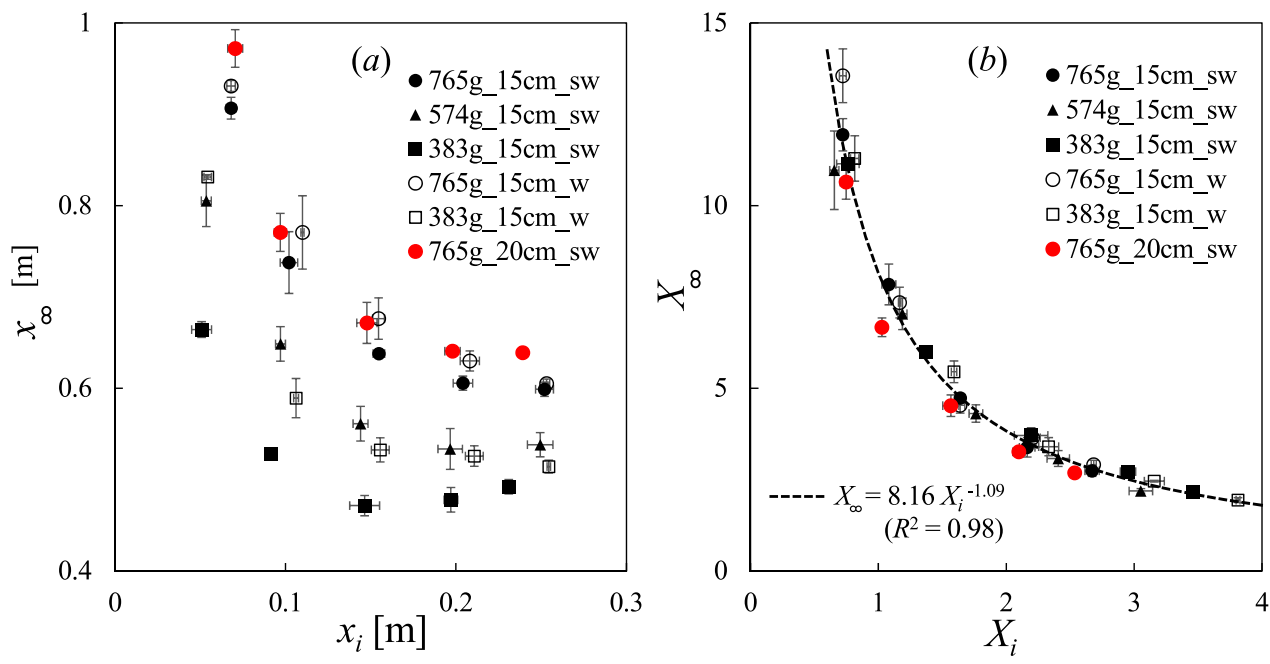

FIgURE 12. The final runout distance $x_{\infty}:(a)$ raw data and $(b)$ rescaled data. The definition of the runout distance $x_{\infty}$ is shown in figure $6 a$. The dashed curve is a best power-law fit. The dimensionless runout distance and lock length are is defined as $X_{\infty} \equiv x_{\infty} / l_{\infty}$ and $X_{i} \equiv x_{i} / l_{c}$, respectively, with $l_{\infty}$ and $l_{c}$ defined in (3.7) and (3.1).

We also note that the collapse of data measuring the front from the lock gate does not collapse as well.

\subsection{Runout distance $x_{\infty}$}

The final runout distance $x_{\infty}$ is shown in figure $12 a$. For short lock lengths $x_{\infty}$ decreases with increasing $x_{i}$, but is almost independent of lock length for $x_{i}>15 \mathrm{~cm}$. There is only a small difference between the fresh and salt water data, but $x_{\infty}$ increases with $m$. Since the extension of the current occurs during the inertial spreading phase, a runout length scale $l_{\infty}$ can be defined as

$$
l_{\infty} \equiv \alpha t_{c}^{\beta}=A l_{c}=A\left[\frac{m}{\rho w_{t}(1-\phi)}\right]^{\frac{1}{2}} .
$$

We define a dimensionless runout distance $X_{\infty} \equiv x_{\infty} / l_{\infty}$, and plot $X_{\infty}$ as a function of the dimensionless lock length $X_{i}$ in figure $12 b$. This scaling collapses the data and shows that $X_{\infty}$ increases with the initial aspect ratio of the bead configuration, and is independent of the buoyancy of the beads. The best power-law fit, shown as the dashed curve in figure $12 b$, is given by

$$
X_{\infty}=8.16 X_{i}^{-1.09}, \quad \text { for } 0.6 \leqslant X_{i} \leqslant 3.8,
$$

with $R^{2}=0.98$. Other data fitting results, using different functional forms of $X_{\infty}$ vs $X_{i}$, are also provided in Appendix B.

Although viscous and surface tension effects may be expected to become important when the spreading rate is slow as the current approaches its final arrested position, collapse of the data shown in figure $12 b$ suggests that the runout distance $x_{\infty}$ is determined during the inertial-dominated period. We also note that the two dimensional effects, i.e., the irregularity of the propagating front across the channel width are larger with increasing lock length $x_{i}$, decreasing $m$, and decreasing buoyancy $\Delta \rho g$. 

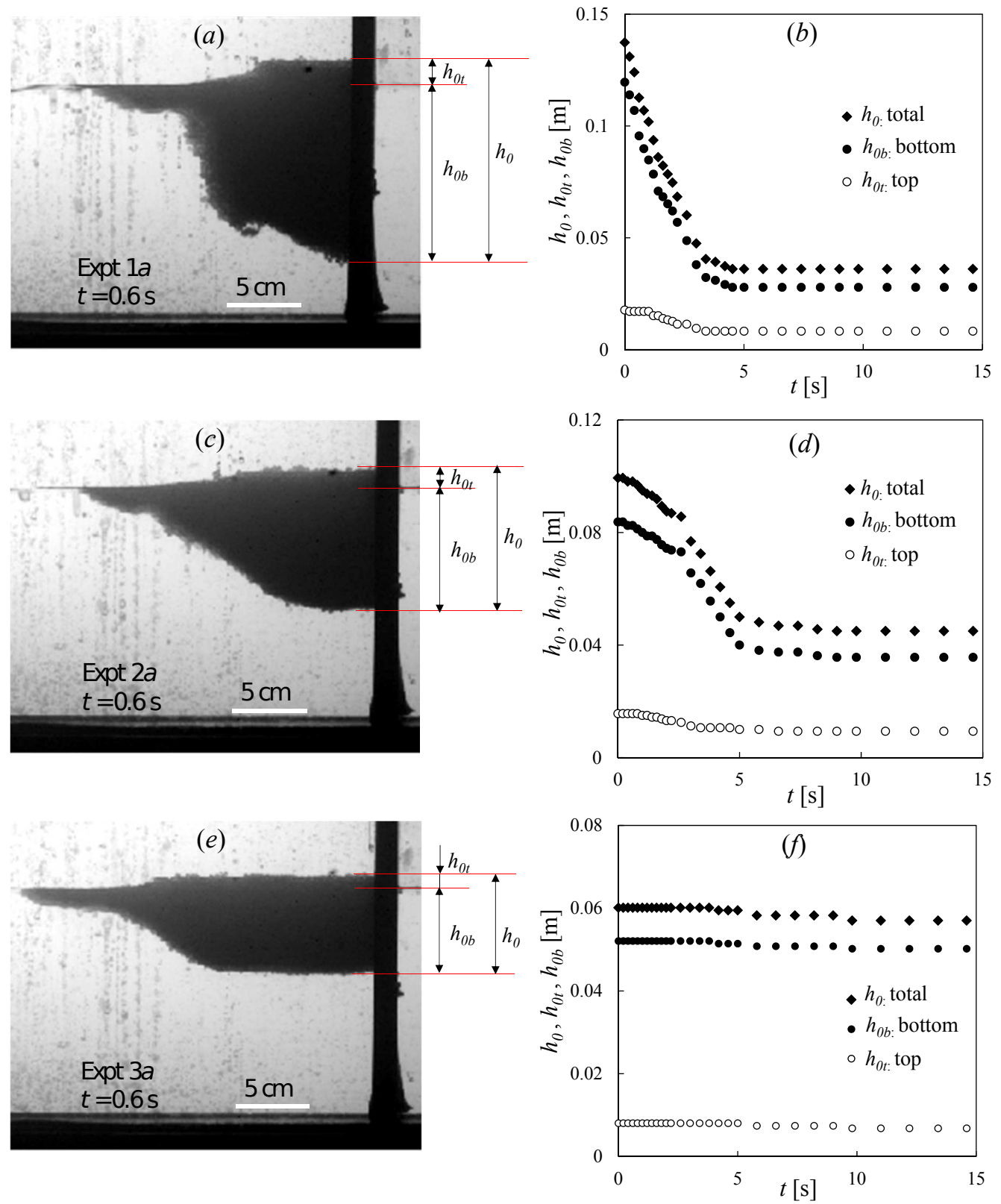

Figure 13. $(a, c, e)$ Profile shapes at $t=0.6 \mathrm{~s}$ for three representative experiments, and the definition of the maximum thicknesses $h_{0 t}, h_{0 b}$, and $h_{0}$. $(b, d, f)$ Time evolution of the corresponding $h_{0 t}, h_{0 b}$. The tank was filled with saturated salt water of depth $d=15 \pm 0.1 \mathrm{~cm}, m=765 \pm 1$ $\mathrm{g}$, and $(a, b) x_{i}=10 \mathrm{~cm},(c, d) x_{i}=15 \mathrm{~cm}$, and $(e, f) x_{i}=20 \mathrm{~cm}$.

\subsection{Maximum thicknesses}

The time evolution of the maximum thicknesses, defined at the back wall of the lock as the distances between the water level and the top surface $h_{0 t}$ and the bottom surface $h_{0 b}$ of the beads, respectively, are shown in figure 13. For small lock lengths $x_{i}<15 \mathrm{~cm}$ (e.g. Expt $1 a$ and Expt $2 a$ ) both $h_{0 t}$ and $h_{b t}$ initially decreased and then became constant as 

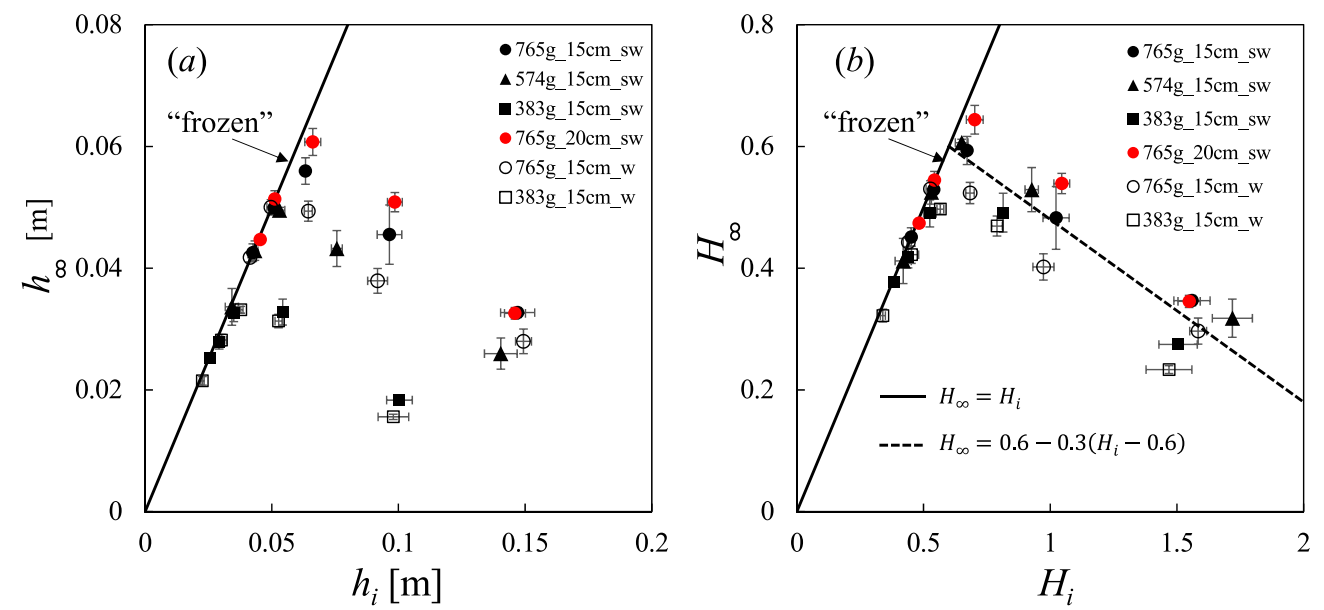

FIGURE 14. The maximum thickness $h_{\infty} \equiv h_{0}(t \rightarrow \infty)$ versus the initial height $h_{i} \equiv h_{0}(t=0)$ of the spreading beads at the origin $x=0$ : $(a)$ raw data and $(b)$ rescaled data. In $(b)$ the dimensionless thicknesses are defined as $H_{\infty} \equiv h_{\infty} / l_{c}$ and $H_{i} \equiv h_{i} / l_{c}$, respectively. The solid line in the figure indicates that the maximum thickness $h_{0}$ remains unchanged during the experiments with $0.3 \leqslant H_{i} \leqslant 0.6$, which corresponds to the existence of a "frozen" region near the origin as shown in figure 5 . The dashed line is given by (3.9).

the current approached its final arrested state. For longer lock length $x_{i} \geqslant 20 \mathrm{~cm}$ (Expt $3 a$ ) both $h_{0 t}$ and $h_{0 b}$ remained close to their initial values, which corresponds to the existence of the "frozen" region near the origin, as shown in figure 5.

The final thickness $h_{\infty} \equiv h_{0}(t \rightarrow \infty)$ at the rear wall of the lock is plotted in figure $14 a$ as a function of initial height $h_{i} \equiv h_{0}(t=0)$ (figure 13). The solid line in the figure indicates the limit that $h_{0}$ remains unchanged during an experiment, which corresponds to the existence of a near origin "frozen" regime, as shown in figure 5.

The final thickness shows a systematic increase with the mass of beads $m$ (figure 14a). In order to account for this we define dimensionless final thickness as $H_{\infty} \equiv h_{\infty} / l_{c}$ and initial height as $H_{i} \equiv h_{i} / l_{c}=1 / X_{i}$, and plot the dimensionless $H_{\infty}$ versus $H_{i}$ in figure $14 b$. This reduces the variation associated with different $m$, and a universal curve of $H_{\infty}$ vs $H_{i}$ is obtained, which can be approximated by

$$
H_{\infty}= \begin{cases}H_{i}, & 0.3 \leqslant H_{i} \leqslant 0.6 \\ 0.6-0.3\left(H_{i}-0.6\right), & 0.6 \leqslant H_{i} \leqslant 1.7 .\end{cases}
$$

This relationship is plotted in figure $14 b$. The solid line indicates that the maximum thickness $H_{0}$ remains unchanged when $0.3 \leqslant H_{i} \leqslant 0.6\left(1.7 \leqslant X_{i} \leqslant 3.8\right)$, while the dashed line provides a reasonable approximate for $H_{\infty}$ for $0.6 \leqslant H_{i} \leqslant 1.7\left(0.6 \leqslant X_{i} \leqslant 1.7\right)$. There appears to be a weaker but systematic buoyancy effect, i.e., an increase in $H_{\infty}$ with $\Delta \rho g$, which is not captured by the current scaling.

More details of the experiments are provided in Appendix A. The Reynolds numbers Re based on the current thickness or an individual bead were $R e \gg 1$ during the intermediate time period (e.g., $1 \mathrm{~s} \leqslant t \leqslant 5 \mathrm{~s}$ ), when the front propagates according to a power-law behaviour, indicating that the current is inertial during this phase. Also, motivated by the front constraining condition of a high Reynolds number gravity current, we computed the Froude number $\mathrm{Fr}$ for the experiments, e.g., based on the bead layer thickness at the origin, and we found that, after a short initial transition (e.g., 1 s), $F r$ decreases as 


\begin{tabular}{|c|c|c|c|c|}
\hline Variables & Physical Meaning & \multicolumn{2}{|c|}{ Representative Experimental Results* } & Reference \\
\hline$\beta$ & scaling exponent & $\beta=0.581 X_{i}^{-0.769}$, & for $0.6 \leqslant X_{i} \leqslant 3.8$ & figure 9 \\
\hline$A$ & prefactor & $A=0.645 e^{0.491 X_{i}}$ & for $0.6 \leqslant X_{i} \leqslant 3.8$ & figure 10 \\
\hline$X_{\infty}$ & runout distance & $X_{\infty}=8.16 X_{i}^{-1.09}$ & for $0.6 \leqslant X_{i} \leqslant 3.8$ & figure 12 \\
\hline$H_{\infty}$ & maximum thickness & $\begin{array}{l}H_{\infty}=H_{i} \\
H_{\infty}=0.6-0.3(H\end{array}$ & $\begin{array}{l}\text { for } 0.3 \leqslant H_{i} \leqslant 0.6 \\
\text { for } 0.6 \leqslant H_{i} \leqslant 1.7\end{array}$ & figure 14 \\
\hline
\end{tabular}

TABLE 1. Summary of the major findings for the time-dependent non-dimensional front location $X_{f}$, the runout distance $X_{\infty}$, and the final thickness at the origin $H_{\infty}$. We note that some of the fitted powers are close to rational fractions. ${ }^{*}$ We only show a respresentative set of data-fitting results that can be used to describe the universal behaviour observed in the experiments. In principle, the experimental data can be fitted using different functional forms, as shown in figure 21 and in Appendix B.

time progresses, and $F r<0.5$ during the majority of the time evolution. This suggests that the condition for an inertial current, that the frontal Froude number is constant and of order one (see, e.g., Simpson 1982) is not appropriate in this situation. In addition, based on the side-view pictures, we calculated the time evolution of the intruding area $A_{i}$, measured between the and the propagating front $x_{f}(t)$. We observe that $A_{i}$ first increases, following the removal of the lock gate, and gradually reaches a constant at long times (e.g., $t=30 \mathrm{~s}$ ). However, to within fairly coarse resolution we are unable to constrain any variations in the mean porosity of the current, and therefore cannot speculate further as to the evolution of the effective rheology of the current during the course of the experiments.

\section{Summary and Discussions}

\subsection{Summary}

In this paper, we investigated the dynamics of a current of spreading beads upon release from behind a lock gate into a liquid of greater density. There are few previous studies on such granular flows despite a rich set of related geophysical and environmental phenomena such as debris flows, industrial waste outputs and volcanic ash spreading. We focused on the time evolution of the front location and the interface shape between the current of beads and the ambient liquid and provided a systematic experimental exploration on the effects of lock length, total amount of beads, and the density of the ambient liquids. We presented scaling arguments to address the major physics that controls the timedependent front location, the finite runout distance and maximum thickness of the bead layer. The rescaled experimental data collapse to universal curves, which can be used to 


\begin{tabular}{|c|c|c|c|}
\hline Category & Runout Distance $x_{\infty}$ & Final Thickness $h_{\infty}$ & Reference \\
\hline Dry collapse & $\frac{x_{\infty}-x_{i}}{x_{i}} \propto \begin{cases}a, & a \leqslant 1.8 \\
a^{2 / 3}, & a \geqslant 2.8\end{cases}$ & $\frac{h_{\infty}}{x_{i}} \propto \begin{cases}a, & a \leqslant 1.15 \\
a^{2 / 5}, & a \geqslant 1.15 .\end{cases}$ & $\begin{array}{l}\text { Lube, et al } \\
\quad(2005)\end{array}$ \\
\hline Dry collapse & $\frac{x_{\infty}-x_{i}}{x_{i}} \propto \begin{cases}a, & a \leqslant 3 \\
a^{1 / 2}, & a \geqslant 3 .\end{cases}$ & $\frac{h_{\infty}}{x_{i}} \propto \begin{cases}a, & a \leqslant 0.7 \\
a^{1 / 3}, & a \geqslant 0.7\end{cases}$ & $\begin{array}{l}\text { Lajeunesse, } \\
\text { et al (2005) }\end{array}$ \\
\hline Dry collapse & $\frac{x_{\infty}-x_{i}}{x_{i}} \propto\left\{\begin{array}{l}a^{0.9}, \text { wide slot } \\
a^{0.65}, \text { narrow slot. }\end{array}\right.$ & $\frac{h_{i}}{h_{\infty}} \propto\left\{\begin{array}{l}a^{0.6}, \text { wide slot } \\
a^{0.5}, \text { narrow slot. }\end{array}\right.$ & $\begin{array}{l}\text { Balmforth, } \\
\text { et al (2005) }\end{array}$ \\
\hline Interfacial intrusion & $\begin{array}{c}\frac{x_{\infty}}{x_{i}} \approx 8.73 a^{0.633} \\
0.07 \leqslant a \leqslant 2.8\end{array}$ & $\frac{h_{\infty}}{x_{i}} \approx\left\{\begin{array}{r}a, \quad 0.07 \leqslant a \leqslant 0.36 \\
a^{1 / 2}\left(0.78-0.3 a^{1 / 2}\right) \\
0.36 \leqslant a \leqslant 2.8\end{array}\right.$ & $\begin{array}{l}\text { current } \\
\text { study }\end{array}$ \\
\hline
\end{tabular}

TABLE 2. A summary of previous and current experimental measurements on the runout distance $x_{\infty}$ and final thickness at the origin $h_{\infty}$ in dry granular collapse and granular intrusion problems. Here $a \equiv h_{i} / x_{i}$, which represents the initial aspect ratio. Note we have rewritten the experimental results of the current study in terms of the aspect ratio in the table, and we have used $A \approx 1.07 X_{i}^{0.824}$ (Appendix B) instead of $A \approx 0.926 e^{-0.455 X_{i}}$ for the runout distance.

describe the dynamic behaviour of these flows. The major findings are summarized in table 1 . We note that some of the fitted powers are close to rational fractions.

While we provide experimental and scaling results for the front dynamics, further theoretical work is necessary to investigate the detailed functional forms of the dependence of the scaling exponent $\beta$, the prefactor $A$, the runout distance $X_{\infty}$ and the maximum thickness $H_{\infty}$ on the initial lock length $X_{i}$, respectively. Theoretical work is also needed to address the time evolution of the profile shape of the current of beads, the packing efficiency, and the existence of the irregular front patterns (figure 3), the shear-driven instability (figure 4), and the "frozen" region (figure 5) in different flow situations.

\subsection{Discussion}

The study has shed light on a category of problems that address the flow of granular materials along a free surface of a liquid, and is expected to motivate further research in the future. Before we close the paper, it is worthwhile to note some major differences between this flow and three other related phenomena: dry and immersed granular collapses in liquids of lower density, the intrusive gravity current, and the particle-laden gravity current. We provide a brief summary in this section.

- A granular density current along a free surface differs in a number of aspects from the dry granular collapse or immersed granular collapse in liquids of lower density. For example, there is no basal friction in the flow along the free surface, although surface tension may play a role, particularly as the current comes to rest. The runout distance of the experiments in this study can be estimated as the maximum horizontal extension of the intermediate period (3.7), during which the location of the spreading front follows 

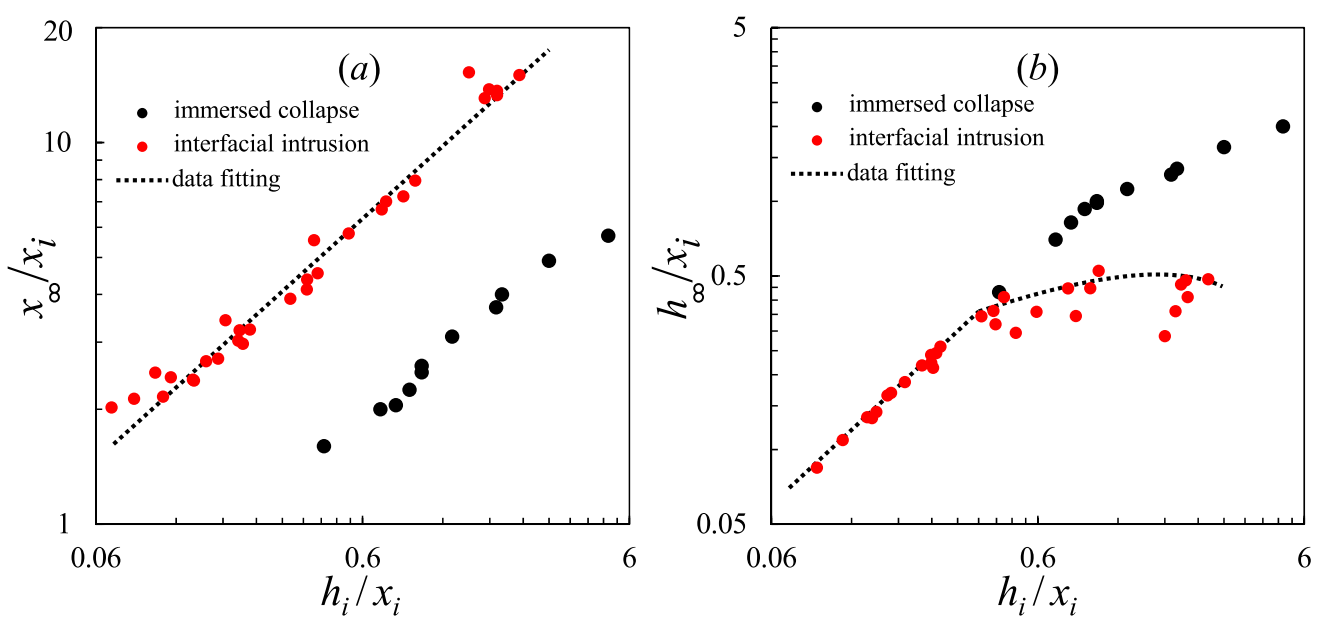

FIGURE 15. Experimental measurements for the $(a)$ runout distance and $(b)$ final thickness for immersed granular collapse (Rondon et al. 2011) and interfacial granular intrusion (current study). The expressions for the fitting curves are shown in table 2.

a power-law time dependence (3.6). However, since the arrested front is only one or two beads thick we expect an increasing role of capillary forces when the granular current slows down. This surface tension may be responsible for the irregular front patterns (figure 3), observed at the end of some of the experiments. In comparison, the granular collapse experiments do not exhibit such behaviours either in dry collapse (Lube et al. 2005; Balmforth \& Kerswell 2005; Lajeunesse et al. 2005) or immersed collapse in a liquid of lower density (Pailha et al. 2008; Rondon et al. 2011). Table 2 summarises the experimental results on the runout distance and final thickness in previous studies of dry granular collapse, which provides a comparison with the current work. In addition, we show in figure 15 the experimental data from immersed granular collapse (from figure 11 in Rondon et al. (2011)) and the current study. The comparison indicates that without basal friction the runout distance in the bead current is greater than that in immersed granular collapse.

- For an intrusive gravity current, the front of the invading fluid continues to propagate, going through an inertial (Holyer \& Huppert 1980; de Rooij et al. 1999) and then a viscous regime (Lister \& Kerr 1989). After an initial brief period of acceleration, the front of the intrusion travels at a constant speed for multiple lock lengths before decelerating (Maxworthy et al. 2002; Maurer et al. 2010), during which time the frontal $\mathrm{Fr}$ is constant and of order one. However, for the granular flows at a free interface, there is no period of constant speed but, instead, the front decelerates and the location of the propagating front follows a power-law time dependence before coming to rest (figures 6-8). Although $R e \gg 1$ during the spreading of the beads (figure 16), Fr decreases monotonically (figure 17), which suggests that the constant $\mathrm{Fr}$ condition is not appropriate during the propagation of the current of beads.

- A granular current along a free surface is also significantly different from the standard particle-laden gravity current. In both flow situations, the buoyant currents reach a finite runout distance. However, for granular current along a free surface, the particles are typically close to each other, and the profile shape of a granular current is set by the envelope of the closely located particles (figure 2), and the size of the beads sets the lower limit for the thickness of the bead layer. In comparison, in particle-laden flows, 
the particles do not pack and are often assumed to distribute homogeneously within the current because of mixing effects. The buoyancy-driven flow eventually stops because the density of the current (an "average" of the particle-fluid system) continues to decrease due to the fall-out of the particles, and the sedimentation speed is often determined by a balance between buoyancy and viscous drag (see, e.g., Bonnecaze et al. 1993).

\section{Acknowledgement}

Z.Z. is grateful for the support of a David Crighton Fellowship from the Department of Applied Mathematics and Theoretical Physics (DAMTP) at the University of Cambridge. J.A.N. is supported by a Royal Society University Research Fellowship. N.M.V. is supported by a Royal Society Dorothy Hodgkin Fellowship. We appreciate the helpful discussions with T. Ball, S. Dalziel, N. O'Keeffe, B. Rallabandi, J. M. F. Tsang, and A. Thomas. We also thank M. Hallworth, D. Page-Croft, and the technicians in the GK Batchelor Laboratory in DAMTP for their help with the set-up of the experimental apparatus.

\section{Appendix A. Additional experimental details}

We provide more details on the experiments in this section. We first summarize the experimental parameters and results in tables 3 and 4 . We calculate the time evolution of the Reynolds number $R e$ and Froude number $F r$ in different flow situations in $\S$ A.1. We also provide the change of effective porosity $\phi_{e}$ and intruding area $A_{i}$ between the location of the lock gate and the front of the bead layer in $\S$ A.2.

\section{A.1. On Reynolds number Re and Froude number Fr}

The time evolution of the Reynolds number Re during the experiments is shown in figure 16. In particular, based on the thickness $h_{i}$ of the bead layer at the location of the lock, $x_{i}$, and the diameter of the beads, $2 r$, we define a Reynolds number by

$$
R e_{i} \equiv \frac{u h_{i}}{\nu}, \quad \text { and } \quad R e_{b} \equiv \frac{2 u r}{\nu},
$$

where $u$ is the speed of the propagating front, and $\nu$ is the kinematic viscosity of the liquid. We note that when the front location follows a power-law behaviour (e.g., $1 \mathrm{~s}$ $\leqslant t \leqslant 5 \mathrm{~s}$ ), the $R e \gg 1$, which suggests that the flow can be considered inviscid during this intermediate period.

We also calculate the time evolution of the Froude number $F r$ in representative experiments. The definition of the $F r$ depends on where we measure the thickness of the current of beads. In particular, since it is difficult to identify a head region and a corresponding thickness near the front of the current of beads, we provide two calculations based on the thickness of beads $h_{i}(t)$ at the location of the lock gate $x=x_{i}$ and the thickness $h_{0}(t)$ at the origin $x=0$, as shown in figure 17. The definitions of the $F r$ are

$$
F r_{i} \equiv \frac{u}{\sqrt{g^{\prime} h_{i}}}, \quad \text { and } \quad F r_{0} \equiv \frac{u}{\sqrt{g^{\prime} h_{0}}},
$$

where $u$ represents the propagating speed of the front of the current of beads and $g^{\prime}$ is the reduced gravity. The representative experiments are identical to those shown in figure 7 and were all conducted in salt water with depth $d=15 \pm 1 \mathrm{~cm}$. The total mass of beads is $m=765 \pm 1 \mathrm{~g}$, while the lock length was varied as $x_{i} \approx\{5,10,15,20,25\} \mathrm{cm}$. The calculation indicates that after a short initial transition (e.g., 1 s), the $F r$ decreases 


\begin{tabular}{|c|c|c|c|c|c|}
\hline Experiment & Liquid Type & $\begin{array}{c}\text { Fluid Density } \\
\rho_{l}\left[10^{3} \mathrm{~kg} \cdot \mathrm{m}^{-3}\right]\end{array}$ & $\begin{array}{l}\text { Fluid Depth } \\
\quad d[\mathrm{~m}]\end{array}$ & $\begin{array}{c}\text { Beads Mass } \\
w_{t}[\mathrm{~kg}]\end{array}$ & $\begin{array}{c}\text { Lock Length } \\
x_{i}[\mathrm{~m}]\end{array}$ \\
\hline $1 a, b, c$ & salt water & 1.18 & 0.015 & 0.765 & $0.066 / 0.072 / 0.066$ \\
\hline $2 \mathrm{a}, \mathrm{b}, \mathrm{c}$ & salt water & 1.18 & 0.015 & 0.765 & $0.101 / 0.109 / 0.096$ \\
\hline $3 a, b, c$ & salt water & 1.18 & 0.015 & 0.765 & $0.159 / 0.151 / 0.154$ \\
\hline $4 a, b, c$ & salt water & 1.18 & 0.015 & 0.765 & $0.208 / 0.208 / 0.196$ \\
\hline $5 \mathrm{a}, \mathrm{b}, \mathrm{c}$ & salt water & 1.18 & 0.015 & 0.765 & $0.257 / 0.254 / 0.245$ \\
\hline $6 \mathrm{a}, \mathrm{b}, \mathrm{c}$ & salt water & 1.18 & 0.015 & 0.574 & $0.052 / 0.058 / 0.051$ \\
\hline $7 \mathrm{a}, \mathrm{b}, \mathrm{c}$ & salt water & 1.18 & 0.015 & 0.574 & $0.100 / 0.093 / 0.099$ \\
\hline $8 a, b, c$ & salt water & 1.18 & 0.015 & 0.574 & $0.150 / 0.142 / 0.140$ \\
\hline $9 \mathrm{a}, \mathrm{b}, \mathrm{c}$ & salt water & 1.18 & 0.015 & 0.574 & $0.195 / 0.189 / 0.206$ \\
\hline $10 \mathrm{a}, \mathrm{b}, \mathrm{c}$ & salt water & 1.18 & 0.015 & 0.574 & $0.239 / 0.257 / 0.252$ \\
\hline $11 \mathrm{a}, \mathrm{b}, \mathrm{c}$ & salt water & 1.18 & 0.015 & 0.383 & $0.055 / 0.043 / 0.055$ \\
\hline $12 \mathrm{a}, \mathrm{b}, \mathrm{c}$ & salt water & 1.18 & 0.015 & 0.383 & $0.090 / 0.090 / 0.094$ \\
\hline $13 a, b, c$ & salt water & 1.18 & 0.015 & 0.383 & $0.159 / 0.140 / 0.141$ \\
\hline $14 \mathrm{a}, \mathrm{b}, \mathrm{c}$ & salt water & 1.18 & 0.015 & 0.383 & $0.193 / 0.196 / 0.202$ \\
\hline $15 \mathrm{a}, \mathrm{b}, \mathrm{c}$ & salt water & 1.18 & 0.015 & 0.383 & $0.233 / 0.228 / 0.232$ \\
\hline $16 \mathrm{a}, \mathrm{b}, \mathrm{c}$ & salt water & 1.18 & 0.020 & 0.765 & $0.065 / 0.070 / 0.076$ \\
\hline $17 \mathrm{a}, \mathrm{b}, \mathrm{c}$ & salt water & 1.18 & 0.020 & 0.765 & $0.096 / 0.102 / 0.093$ \\
\hline $18 \mathrm{a}, \mathrm{b}, \mathrm{c}$ & salt water & 1.18 & 0.020 & 0.765 & $0.142 / 0.156 / 0.145$ \\
\hline $19 \mathrm{a}, \mathrm{b}, \mathrm{c}$ & salt water & 1.18 & 0.020 & 0.765 & $0.192 / 0.203 / 0.199$ \\
\hline $20 a, b, c$ & salt water & 1.18 & 0.020 & 0.765 & $0.240 / 0.237 / 0.240$ \\
\hline $21 \mathrm{a}, \mathrm{b}$ & fresh water & 1.0 & 0.015 & 0.765 & $0.071 / 0.066$ \\
\hline $22 \mathrm{a}, \mathrm{b}, \mathrm{c}$ & fresh water & 1.0 & 0.015 & 0.765 & $0.111 / 0.109 / 0.111$ \\
\hline $23 \mathrm{a}, \mathrm{b}, \mathrm{c}$ & fresh water & 1.0 & 0.015 & 0.765 & $0.150 / 0.156 / 0.158$ \\
\hline $24 \mathrm{a}, \mathrm{b}$ & fresh water & 1.0 & 0.015 & 0.765 & $0.214 / 0.202$ \\
\hline $25 \mathrm{a}, \mathrm{b}$ & fresh water & 1.0 & 0.015 & 0.765 & $0.255 / 0.251$ \\
\hline $26 \mathrm{a}, \mathrm{b}, \mathrm{c}$ & fresh water & 1.0 & 0.015 & 0.383 & $0.054 / 0.052 / 0.057$ \\
\hline $27 \mathrm{a}, \mathrm{b}, \mathrm{c}$ & fresh water & 1.0 & 0.015 & 0.383 & $0.108 / 0.104 / 0.107$ \\
\hline $28 \mathrm{a}, \mathrm{b}, \mathrm{c}$ & fresh water & 1.0 & 0.015 & 0.383 & $0.158 / 0.161 / 0.149$ \\
\hline $29 \mathrm{a}, \mathrm{b}, \mathrm{c}$ & fresh water & 1.0 & 0.015 & 0.383 & $0.216 / 0.213 / 0.204$ \\
\hline $30 a, b, c$ & fresh water & 1.0 & 0.015 & 0.383 & $0.256 / 0.253 / 0.254$ \\
\hline
\end{tabular}

TABLE 3. Summary of the experimental parameters. All experiments were conducted in a rectangular tank of length $l_{t}=2.09 \pm 0.01 \mathrm{~m}$, width $w_{t}=0.15 \pm 0.01 \mathrm{~m}$, and depth $d_{t}=0.57 \pm 0.01$ m. Either saturated salt water (saturated $\mathrm{NaCl}$ solution), with density $\rho_{s w}=1.18 \times 10^{3} \mathrm{~kg} \mathrm{~m}^{-3}$, or fresh water, with density $\rho_{w}=1.0 \times 10^{3} \mathrm{~kg} \mathrm{~m}^{-3}$, was used in the experiments. The depth of the liquid bath $d$, the total mass of the beads $m$, and the lock length $x_{i}$ were also varied, as listed in the table. Spherical polypropylene beads (McMaster Carr, 1974K2) were used in all the experiments with a radius $r=1.59 \mathrm{~mm}$ and density $\rho=0.91 \times 10^{3} \mathrm{~kg} / \mathrm{m}^{3}$, measured at room temperature. Experiments with similar parameters were conducted to test the reproducibility. 


\begin{tabular}{|c|c|c|c|c|c|}
\hline Experiment & $\begin{array}{l}\text { Scaling } \\
\text { Exponent } \\
\beta\end{array}$ & $\begin{array}{l}\text { Prefactor } \\
\alpha\left[\mathrm{m} / \mathrm{s}^{\beta}\right]\end{array}$ & $\begin{array}{l}\text { Runout } \\
\text { Distance } \\
x_{\infty}[\mathrm{m}]\end{array}$ & $\begin{array}{l}\text { Initial } \\
\text { Thickness } \\
h_{i}[\mathrm{~m}]\end{array}$ & $\begin{array}{l}\text { Final } \\
\text { Thickness } \\
h_{\infty}[\mathrm{m}]\end{array}$ \\
\hline 1a,b,c & $0.716 \pm 0.014$ & $0.239 \pm 0.004$ & $0.907 \pm 0.012$ & $0.147 \pm 0.007$ & $0.033 \pm 0.001$ \\
\hline $2 a, b, c$ & $0.584 \pm 0.017$ & $0.237 \pm 0.001$ & $0.738 \pm 0.034$ & $0.096 \pm 0.005$ & $0.046 \pm 0.005$ \\
\hline $3 a, b, c$ & $0.417 \pm 0.003$ & $0.254 \pm 0.003$ & $0.638 \pm 0.003$ & $0.063 \pm 0.001$ & $0.056 \pm 0.002$ \\
\hline $4 a, b, c$ & $0.327 \pm 0.030$ & $0.282 \pm 0.007$ & $0.606 \pm 0.008$ & $0.051 \pm 0.001$ & $0.050 \pm 0.001$ \\
\hline $5 \mathrm{a}, \mathrm{b}, \mathrm{c}$ & $0.272 \pm 0.023$ & $0.314 \pm 0.009$ & $0.599 \pm 0.008$ & $0.043 \pm 0.001$ & $0.043 \pm 0.001$ \\
\hline $6 a, b, c$ & $0.690 \pm 0.028$ & $0.231 \pm 0.005$ & $0.805 \pm 0.028$ & $0.140 \pm 0.006$ & $0.026 \pm 0.003$ \\
\hline $7 a, b, c$ & $0.539 \pm 0.019$ & $0.225 \pm 0.004$ & $0.649 \pm 0.019$ & $0.076 \pm 0.002$ & $0.043 \pm 0.003$ \\
\hline $8 a, b, c$ & $0.401 \pm 0.015$ & $0.253 \pm 0.002$ & $0.561 \pm 0.019$ & $0.053 \pm 0.002$ & $0.050 \pm 0.005$ \\
\hline $9 a, b, c$ & $0.314 \pm 0.014$ & $0.292 \pm 0.002$ & $0.534 \pm 0.022$ & $0.043 \pm 0.001$ & $0.043 \pm 0.001$ \\
\hline $10 \mathrm{a}, \mathrm{b}, \mathrm{c}$ & $0.209 \pm 0.011$ & $0.347 \pm 0.007$ & $0.548 \pm 0.013$ & $0.034 \pm 0.003$ & $0.034 \pm 0.003$ \\
\hline $11 \mathrm{a}, \mathrm{b}, \mathrm{c}$ & $0.679 \pm 0.006$ & $0.197 \pm 0.002$ & $0.664 \pm 0.009$ & $0.100 \pm 0.005$ & $0.018 \pm 0.001$ \\
\hline $12 \mathrm{a}, \mathrm{b}, \mathrm{c}$ & $0.470 \pm 0.010$ & $0.201 \pm 0.003$ & $0.528 \pm 0.005$ & $0.054 \pm 0.001$ & $0.033 \pm 0.002$ \\
\hline $13 \mathrm{a}, \mathrm{b}, \mathrm{c}$ & $0.343 \pm 0.022$ & $0.233 \pm 0.001$ & $0.472 \pm 0.011$ & $0.035 \pm 0.002$ & $0.033 \pm 0.001$ \\
\hline $14 \mathrm{a}, \mathrm{b}, \mathrm{c}$ & $0.274 \pm 0.018$ & $0.284 \pm 0.006$ & $0.478 \pm 0.013$ & $0.029 \pm 0.001$ & $0.028 \pm 0.001$ \\
\hline $15 \mathrm{a}, \mathrm{b}, \mathrm{c}$ & $0.200 \pm 0.017$ & $0.323 \pm 0.007$ & $0.492 \pm 0.008$ & $0.026 \pm 0.006$ & $0.025 \pm 0.001$ \\
\hline $16 \mathrm{a}, \mathrm{b}, \mathrm{c}$ & $0.682 \pm 0.018$ & $0.269 \pm 0.004$ & $0.972 \pm 0.021$ & $0.146 \pm 0.004$ & $0.033 \pm 0.001$ \\
\hline $17 \mathrm{a}, \mathrm{b}, \mathrm{c}$ & $0.530 \pm 0.011$ & $0.268 \pm 0.003$ & $0.771 \pm 0.021$ & $0.099 \pm 0.003$ & $0.051 \pm 0.002$ \\
\hline $18 \mathrm{a}, \mathrm{b}, \mathrm{c}$ & $0.402 \pm 0.018$ & $0.281 \pm 0.004$ & $0.672 \pm 0.022$ & $0.066 \pm 0.003$ & $0.061 \pm 0.002$ \\
\hline $19 \mathrm{a}, \mathrm{b}, \mathrm{c}$ & $0.314 \pm 0.003$ & $0.323 \pm 0.006$ & $0.641 \pm 0.001$ & $0.051 \pm 0.002$ & $0.051 \pm 0.001$ \\
\hline $20 \mathrm{a}, \mathrm{b}, \mathrm{c}$ & $0.177 \pm 0.004$ & $0.362 \pm 0.004$ & $0.639 \pm 0.005$ & $0.045 \pm 0.001$ & $0.045 \pm 0.002$ \\
\hline $21 \mathrm{a}, \mathrm{b}$ & $0.779 \pm 0.020$ & $0.164 \pm 0.006$ & $0.931 \pm 0.006$ & $0.149 \pm 0.003$ & $0.028 \pm 0.002$ \\
\hline $22 \mathrm{a}, \mathrm{b}, \mathrm{c}$ & $0.563 \pm 0.014$ & $0.197 \pm 0.004$ & $0.771 \pm 0.040$ & $0.092 \pm 0.004$ & $0.038 \pm 0.002$ \\
\hline $23 \mathrm{a}, \mathrm{b}, \mathrm{c}$ & $0.402 \pm 0.024$ & $0.234 \pm 0.004$ & $0.676 \pm 0.023$ & $0.064 \pm 0.002$ & $0.049 \pm 0.001$ \\
\hline $24 \mathrm{a}, \mathrm{b}$ & $0.362 \pm 0.009$ & $0.259 \pm 0.007$ & $0.630 \pm 0.011$ & $0.050 \pm 0.002$ & $0.050 \pm 0.001$ \\
\hline $25 \mathrm{a}, \mathrm{b}$ & $0.281 \pm 0.008$ & $0.285 \pm 0.012$ & $0.605 \pm 0.005$ & $0.041 \pm 0.001$ & $0.041 \pm 0.001$ \\
\hline $26 \mathrm{a}, \mathrm{b}, \mathrm{c}$ & $0.661 \pm 0.012$ & $0.173 \pm 0.007$ & $0.831 \pm 0.002$ & $0.098 \pm 0.006$ & $0.016 \pm 0.001$ \\
\hline $27 \mathrm{a}, \mathrm{b}, \mathrm{c}$ & $0.451 \pm 0.007$ & $0.194 \pm 0.004$ & $0.589 \pm 0.021$ & $0.053 \pm 0.001$ & $0.031 \pm 0.001$ \\
\hline $28 \mathrm{a}, \mathrm{b}, \mathrm{c}$ & $0.320 \pm 0.014$ & $0.237 \pm 0.008$ & $0.533 \pm 0.013$ & $0.038 \pm 0.001$ & $0.033 \pm 0.001$ \\
\hline $29 \mathrm{a}, \mathrm{b}, \mathrm{c}$ & $0.242 \pm 0.014$ & $0.292 \pm 0.007$ & $0.526 \pm 0.011$ & $0.030 \pm 0.002$ & $0.028 \pm 0.001$ \\
\hline $30 \mathrm{a}, \mathrm{b}, \mathrm{c}$ & $0.174 \pm 0.021$ & $0.332 \pm 0.009$ & $0.514 \pm 0.007$ & $0.023 \pm 0.001$ & $0.021 \pm 0.001$ \\
\hline
\end{tabular}

TABLE 4. Summary of experimental results. The experimental data for the time evolution of the front location are fitted to a power-law form of $x_{f}(t)=\alpha t^{\beta}$ during an intermediate time period. The values of the scaling exponent $\beta$ and the prefactor $\alpha$ from a best least-squares fit of the experimental data are reported. The runout distance $x_{\infty}$ represents the length of the beads at the end of the experiments, once the motion has stopped. The initial thickness represents the thickness of the beads at the origin $x=0$ at the beginning of the experiments, i.e., $h_{i} \equiv h_{0}(t=0)$, while the final thickness $h_{\infty}$ represents the thickness of the beads at $x=0$ at the end of the experiments, i.e., $h_{\infty} \equiv h_{0}(t \rightarrow \infty)$, as shown in figure 13 . 

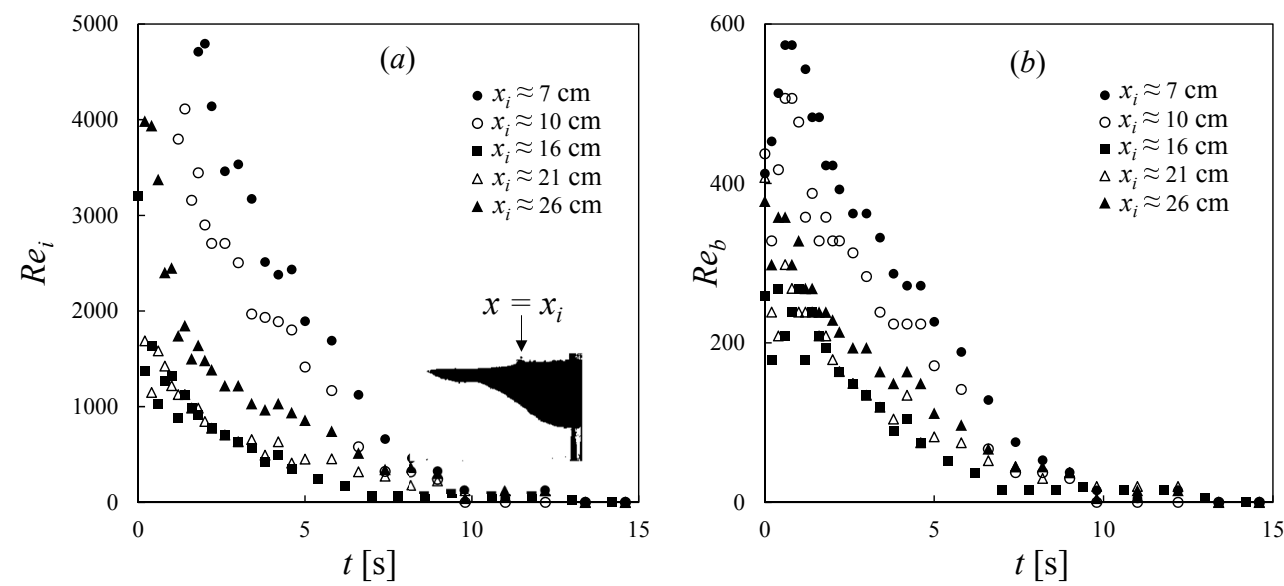

FiguRE 16. The time evolution of $R e$ in representative experiments: $(a) R e_{i} \equiv u h_{i} / \nu$ with $h_{i}$ denoting the thickness of the beads at the location of the lock gate $x_{i}$ and $(b) \operatorname{Re}_{b} \equiv 2 u r / \nu$ based on the diameter of the beads. The experiments are identical to those in figure 7 .
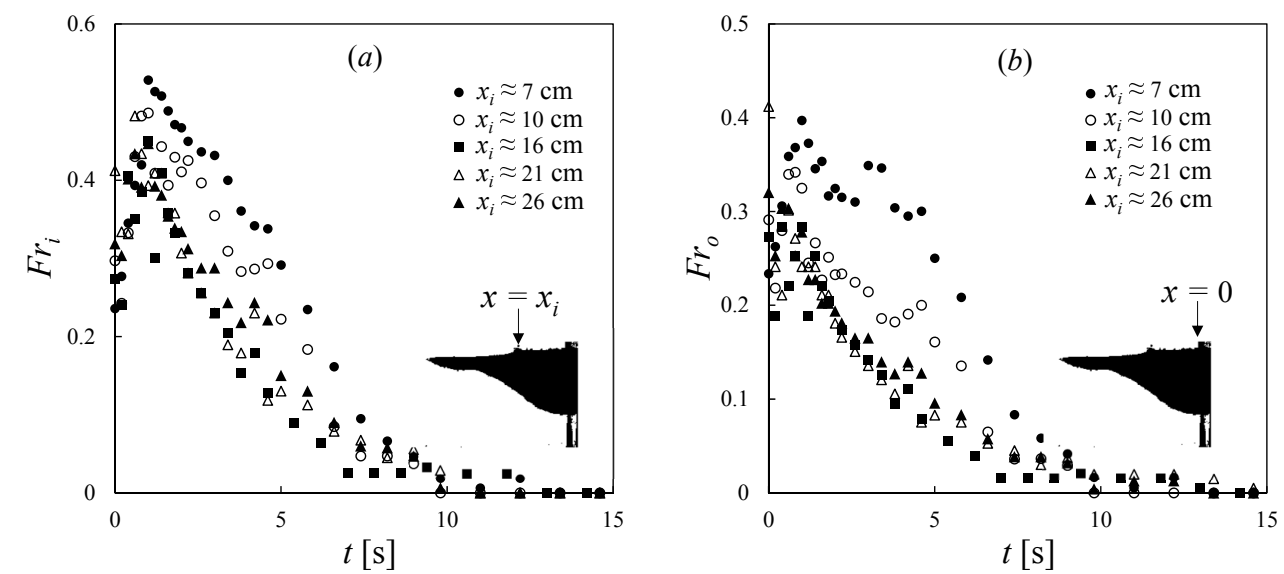

FiguRE 17. The time evolution of the $F r$ in representative experiments: $(a) F r_{i} \equiv u / \sqrt{g^{\prime} h_{i}}$ with $h_{i}$ denoting the thickness of the beads at the location of the lock gate $x_{i}$ and $(b) F r_{0} \equiv u / \sqrt{g^{\prime} h_{0}}$ with $h_{0}$ representing the thickness of the beads at the origin $x=0 . u$ is the propagating speed of the front of the current of beads, and $g^{\prime}$ is the reduced gravity. The experiments are identical to those in figure 7 .

monotonically as time progresses, and $F r<0.5$ during most of the time period. We note that the frontal $\mathrm{Fr}$ is constant and of order one for an inertial current (see, e.g., Simpson 1982), and our calculation suggests that this constant frontal $F r$ condition is not appropriate for the spreading of the current of beads. Generally, we do not expect the Froude number to be a relevant parameter for these flows.

\section{A.2. On porosity $\phi_{e}$ and intruding area $A_{i}$}

We show in figure 18 the incremental change of area covered by the bead layer: the blue region represents an increase in area, and the red region indicates a decrease. As time progresses, the length of the current extends, which is mainly sustained by a decrease in the thickness of the current within the lock region. The difference in the area covered by the blue and red regions indicates a porosity change for the current of beads, which we 

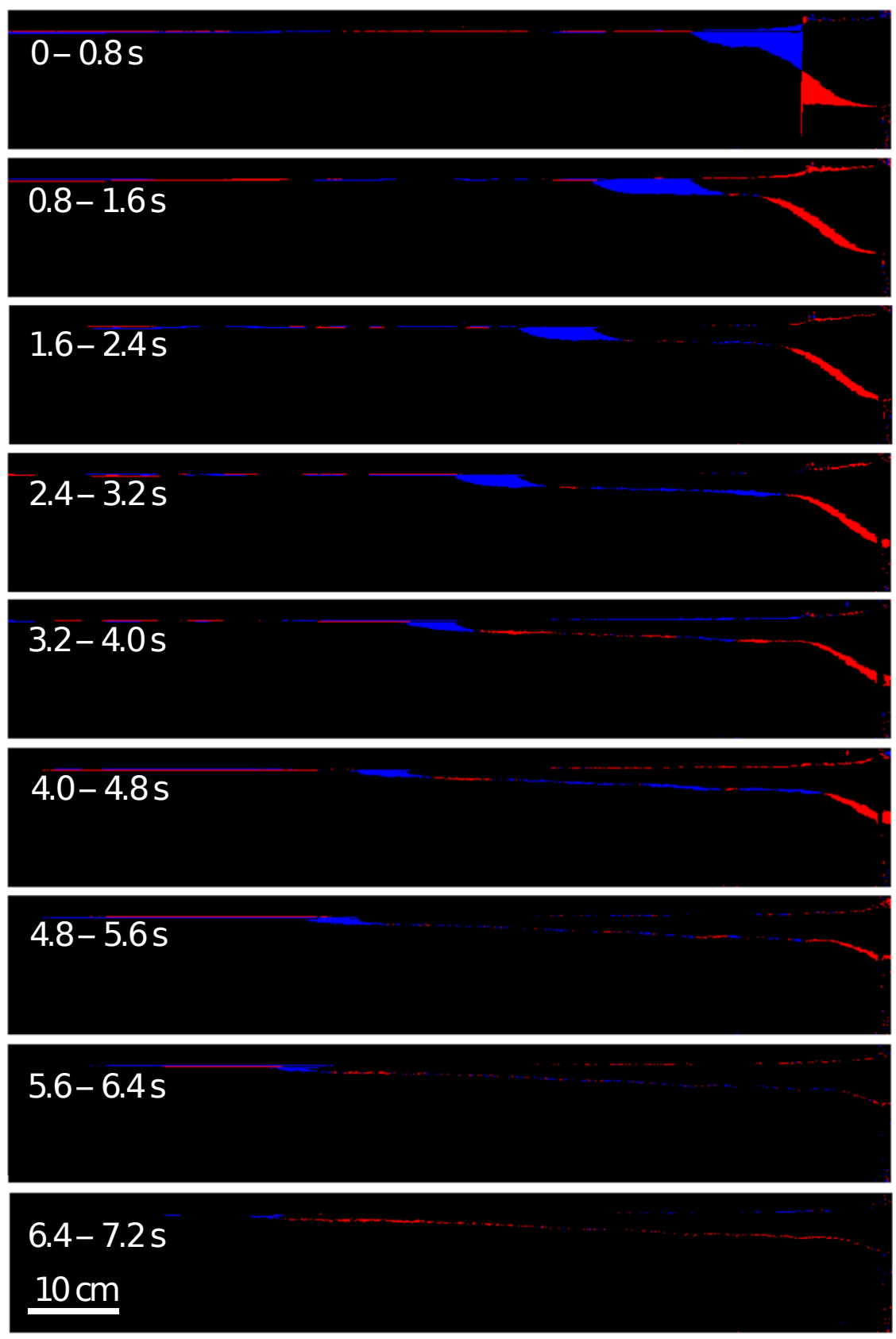

FIGURE 18. The incremental change of area of the bead layer for the experiment shown in figure 2. The blue region represents an increase in area, while the red region indicates a decrease. The downstream signals represent small changes in the free surface height. 

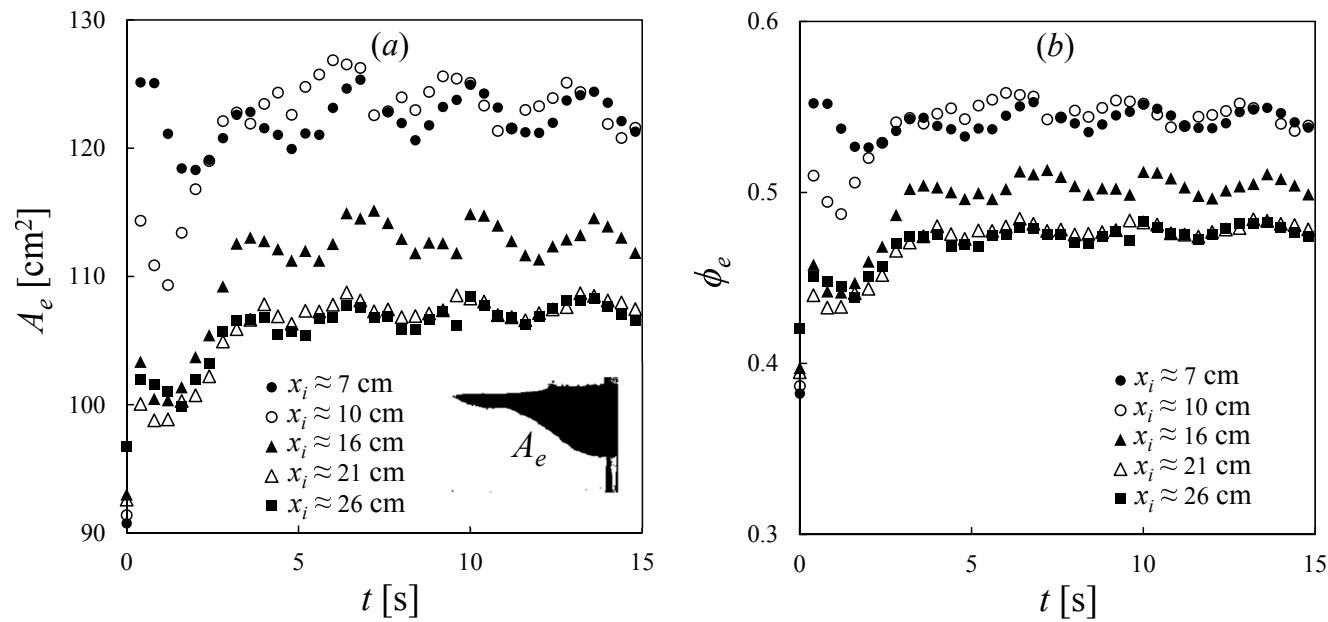

FIGURE 19. Calculations based on side-view pictures in representative experiments: $(a)$ Area $A_{e}$ for the current of beads and $(b)$ Porosity $\phi_{e}$, computed based on $\phi_{e} \equiv 1-m /\left(\rho w_{t} A_{e}\right)$, where $m$ and $\rho$ are the mass and density of the beads, respectively, and $w_{t}$ is the width of the water tank. The experiments are identical to those in figure 17.
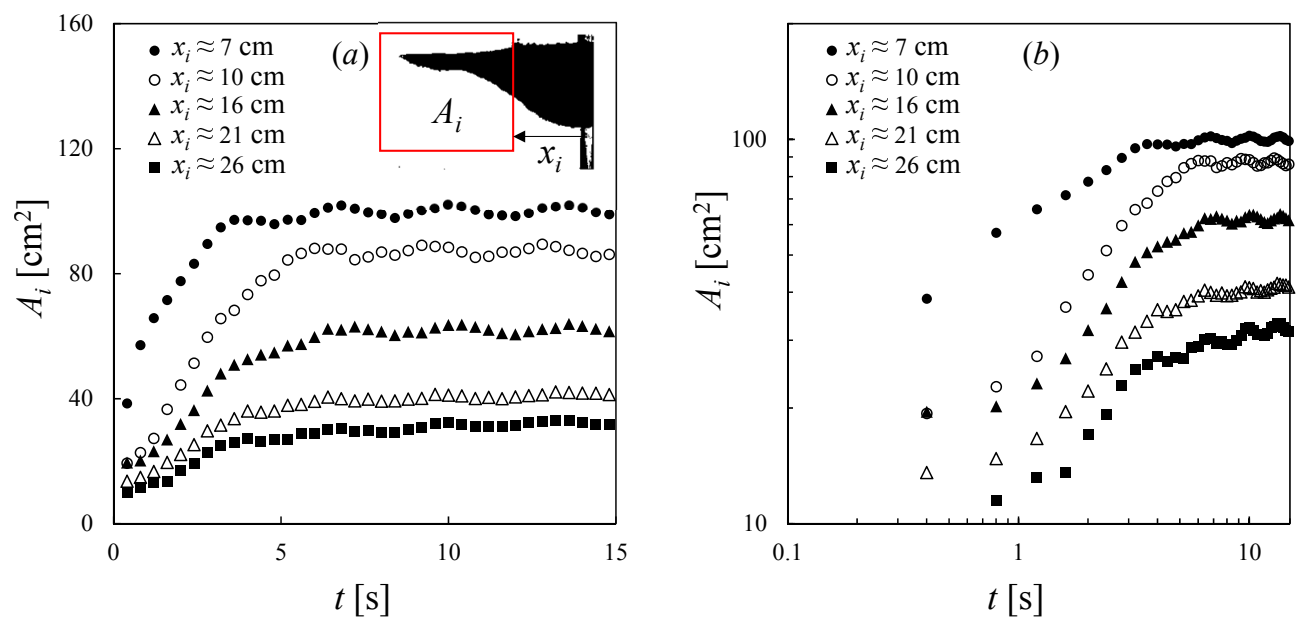

Figure 20. The time evolution of the intruding area $A_{i}(t)$ measured from the location of the lock gate $x_{i}$ to the front $x_{f}(t)$. The definition of the intruding area is shown in the inset of $(a)$, and the experimental results are shown in a log-log graph in $(b)$. The experiments are identical to those in figure 17 .

provide a detailed calculation in this section. Eventually the front of the beads reaches a maximum location, all motion stops and the interface shape stays unchanged at later times.

First, the total area $A_{e}$ can be calculated from the side-view pictures in representative experiments, as shown in figure $19 a$. We observe an increase in $A_{e}$ immediately following the release of beads from the lock gate. We can then compute the effective porosity $\phi_{e}$ for the system of beads based on $\phi_{e} \equiv 1-m /\left(\rho w_{t} A_{e}\right)$. The results are shown in figure $19 b$, where we observe an increase of $\phi_{e}$ following the removal of the lock gate. The increase in $A_{e}$ and $\phi_{e}$ is mainly because the beads are no longer closely packed once they start to spread horizontally. In addition, based on the side-view pictures, we can obtain the time 
evolution of the intruding area $A_{i}$, measured from the location of the lock gate $x_{i}$ to the location of the propagating front $x_{f}(t)$. The results from representative experiments are shown in figure 20, and we observe that $A_{i}$ first increases before gradually reaching a constant value.

We note that the thickness of beads can vary across the width of the water tank, i.e., perpendicular to the plane of the paper, which is an uncertainty in the experimental measurements. In addition, a surface wave (with an amplitude of $\approx 1 \mathrm{~cm}$, for example), generated from pulling the gate out and the impact of beads, can also contribute to the experimental uncertainty. For example, the oscillations in figures 19 and 20 are likely caused by the sloshing water wave. In fact, based on long-wave theory, we estimate that the water wave travels with a speed of $\sqrt{g d} \approx 1.2 \mathrm{~m} / \mathrm{s}$. Thus, the period of the oscillations is estimated to be $2 l_{t} / \sqrt{g d} \approx 3.4 \mathrm{~s}$, which agrees with the experimental observations.

\section{Appendix B. On experimental data fitting}

We note that the experimental data can be fitted using other functional forms, besides those introduced in the main text and summarized in table 1 . Here we provide more data-fitting results for the scaling exponent $\beta$, prefactor $A$, and runout distance $X_{\infty}$ as a function of the rescaled initial lock length $X_{i}$, as also illustrated in figure 21 . The results hold for $0.6 \leqslant X_{i} \leqslant 3.8$, as set by the experiments. We also note that some of the fitted powers are close to rational fractions.

In particular, we provide the best exponential, logarithm, and power-law fitting results for the scaling exponent $\beta$,

$$
\beta= \begin{cases}0.926 e^{-0.455 X_{i}}, & R^{2}=0.971, \\ -0.324 \ln X_{i}+0.591, & R^{2}=0.976, \\ 0.581 X_{i}^{-0.769}, & R^{2}=0.962 .\end{cases}
$$

We also provide the best exponential, linear, and power-law fitting results for the dimensionless prefactor $A$,

$$
A= \begin{cases}0.645 e^{0.491 X_{i}}, & R^{2}=0.947, \\ 0.944 X_{i}, & R^{2}=0.929, \\ 1.07 X_{i}^{0.824}, & R^{2}=0.924 .\end{cases}
$$

In addition, we provide the best exponential, logarithm, and power-law fitting results for the dimensionless runout distance $X_{\infty}$,

$$
X_{\infty}= \begin{cases}15.2 e^{-0.622 X_{i}}, & R^{2}=0.927, \\ -6.21 \ln X_{i}+8.72, & R^{2}=0.919, \\ 8.16 X_{i}^{-1.09}, & R^{2}=0.982 .\end{cases}
$$

The data-fitting results can be used to describe the universal behaviours for the propagating front in the interfacial granular intrusion experiments.

\section{Appendix C. On the spreading time}

We investigate in this section the spreading time $t_{\infty}$ of the current of beads. The definition of the spreading time $t_{\infty}$ is given by $x_{\infty}=\alpha t_{\infty}^{\beta}$. Thus, once $\alpha, \beta$ and $x_{\infty}$ are identified from each experiments, the value of $t_{\infty}$ can be computed. The experimental data are shown in figure $22 a$. The error bars are longer than those of $\alpha, \beta$ and $x_{\infty}$, 

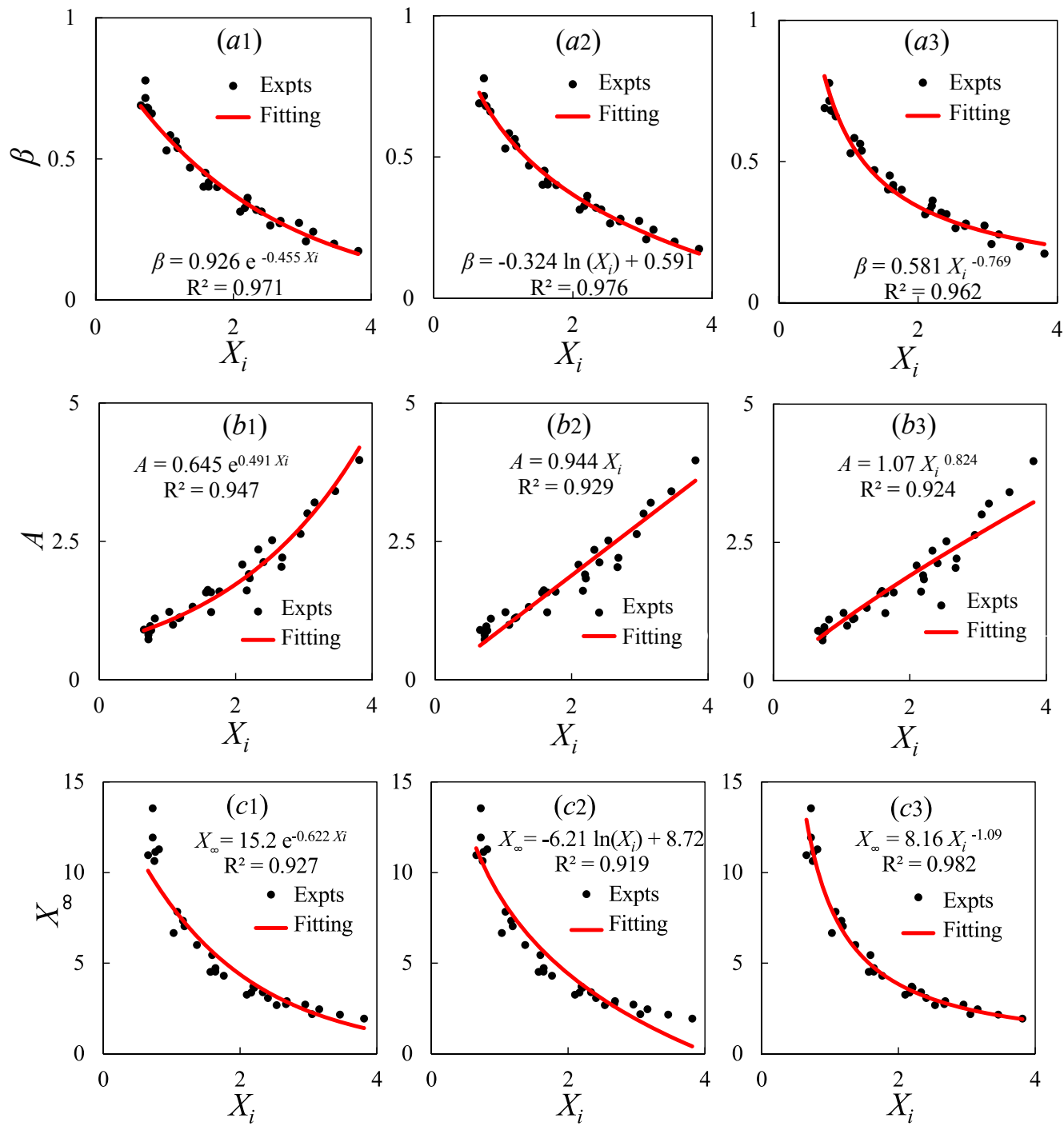

FIGURE 21. Data fitting for the experimental measurements, using different functional forms: (a1-a3) Scaling exponent, (b1-b3) Prefactor, and (c1-c3) Runout distance. The results can be used to describe the universal dynamic behaviours for the front location observed in the interfacial granular intrusion experiments.

especially for fresh water experiments with a large initial lock length $x_{i}$ (e.g., $x_{i} \geqslant 15$ $\mathrm{cm})$.

We can further define the dimensionless spreading time as $T_{\infty} \equiv t_{\infty} / t_{c}$, where the characteristic time $t_{c}$ is defined by (3.5). We observe that the rescaled data collapse to a universal curve, as shown in figure $22 b$, which confirms that $t_{c}$ correctly captures the time scale of the spreading dynamics of the granular intrusion experiments. We also note that the spreading time $t_{\infty}$ is defined based on $\alpha, \beta$ and $x_{\infty}$, and $T_{\infty}\left(X_{i}\right)$ can be estimated based on

$$
T_{\infty}=X_{\infty}^{1 / \beta}
$$

where $\beta\left(X_{i}\right)$ and $X_{\infty}\left(X_{i}\right)$ are provided from (B 1) and (B 3), respectively. The dashed 

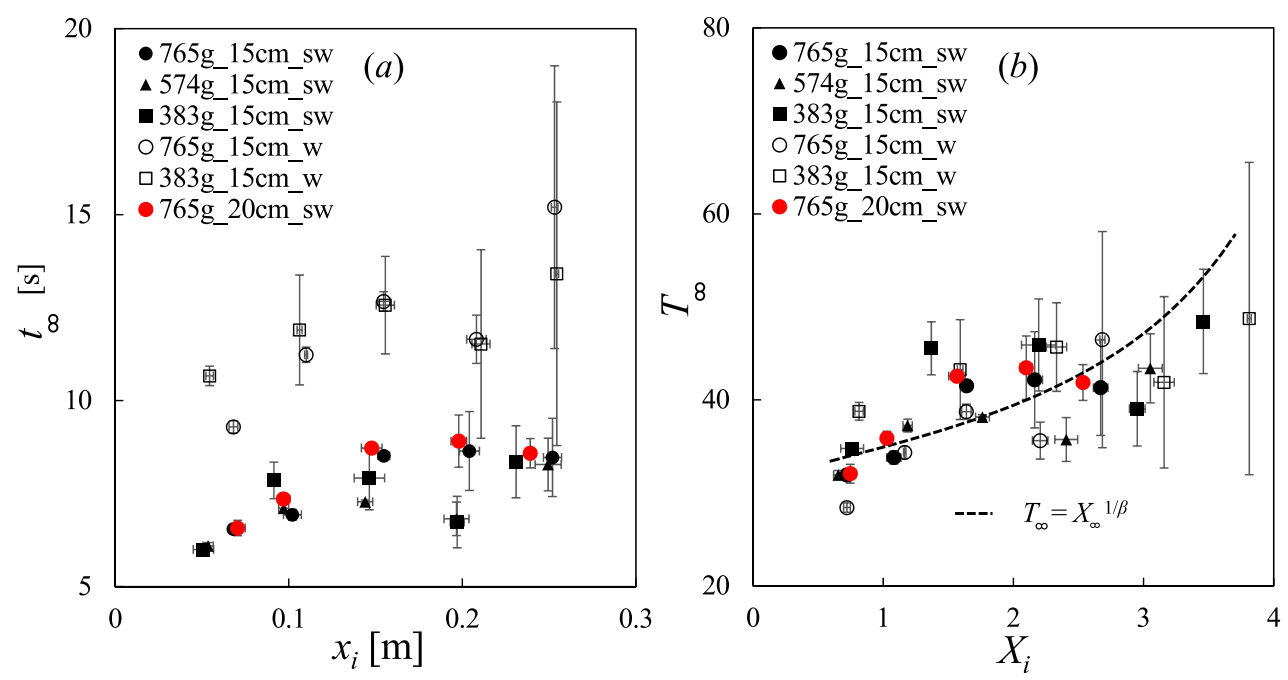

Figure 22. The spreading time $t_{\infty}$ in different experiments: $(a)$ raw data and $(b)$ rescaled data. $t_{\infty}$ is defined based on $x_{\infty}=\alpha t_{\infty}^{\beta}$ and the dashed curve in $(b)$ is based on (B 1b), (B 3c) and $(\mathrm{C} 1)$. The data collapse in the rescaled plot $(b)$ and the agreement with the dashed curve further confirm that the characteristic time and length scales $t_{c}$ and $l_{c}$, defined as (3.5) and (3.1), correctly capture the spreading dynamics of the current of beads.

curve in figure $22 b$ is a sample calculation of $T_{\infty}\left(X_{i}\right)$ based on (B 1b), (B 3c) and (C 1). The agreement with the rescaled experimental data on $T_{\infty}\left(X_{i}\right)$ further confirms the self-consistence of the scaling arguments in this study.

\section{REFERENCES}

Acton, J. M., Huppert, H. E. \& Worster, M. G. 2001 Two-dimensional viscous gravity currents flowing over a deep porous medium. J. Fluid Mech. 440, 359-380.

Balmforth, N. J. \& Kerswell, R. R. 2005 Granular collapse in two dimensions. J. Fluid Mech. 538, 399-428.

Bonnecaze, R. T., Hallworth, M. A., Huppert, H. E. \& Lister, J. R. 1995 Axisymmetric particle-driven gravity currents. J. Fluid Mech. 294, 93-121.

Bonnecaze, R. T., Huppert, H. E. \& Lister, J. R. 1993 Particle-driven gravity currents. J. Fluid Mech. 250, 339-369.

Carazzo, G. \& Jellinek, A. M. 2012 A new view of the dynamics, stability and longevity of volcanic clouds. Earth Planet. Sci. Lett. 325, 39-51.

DAlziel, S. B. 2005 Digiflow user guide. Tech. Rep.. Department of Applied Mathematics and Theoretical Physics, University of Cambridge.

De Rooij, F., Linden, P. F. \& Dalziel, S. B. 1999 Saline and particle-driven interfacial intrusions. J. Fluid Mech. 389, 303-334.

Du Pont, S. CC., Gondret, P., Perrin, B. \& Rabaud, M. 2003 Granular avalanches in fluids. Phys. Rev. Lett. 90, 044301.

Hallworth, M. A. \& Huppert, H. E. 1998 Abrupt transitions in high-concentration, particledriven gravity currents. Phys. Fluids 10, 1083-1087.

Holyer, J. Y. \& Huppert, H. E. 1980 Gravity currents entering a two-layer fluid. J. Fluid Mech. 100, 739-767.

Hoult, D. 1972 Oil spreading in the sea. Ann. Rev. Fluid Mech. 4, 341-368.

Lajeunesse, E., Mangeney-Castelnau, A. \& Vilotte, J. 2004 Spreading of a granular mass on a horizontal plane. Phys. Fluids 16, 2371-2381. 
Lajeunesse, E., Monnier, J. B. \& Homsy, G. M. 2005 Granular slumping on a horizontal surface. Phys. Fluids 17, 103302.

Larrieu, E., Staron, L. \& Hinch, E. J. 2006 Raining into shallow water as a description of the collapse of a column of grains. J. Fluid Mech. 554, 259-270.

Lister, J. R. \& KerR, R. C. 1989 The propagation of two-dimensional and axisymmetric viscous gravity currents at a fluid interface. J. Fluid Mech. 203, 215-249.

Lube, G., Huppert, H. E., Sparks, R. S. J. \& Freundt, A. 2005 Collapses of twodimensional granular columns. Phys. Rev. E 72, 041301.

Lube, G., Huppert, H. E., Sparks, R. S. J. \& Hallworth, M. A. 2004 Axisymmetric collapse of granular columns. J. Fluid Mech. 508, 175-199.

Maurer, B. D., Bolster, D. T. \& Linden, P. F. 2010 Intrusive gravity currents between two stably stratified fluids. J. Fluid Mech. 647, 53-69.

Maxworthy, T., Leilich, J., Simpson, J. E. \& Meiburg, E. H. 2002 The propagation of a gravity current into a linearly stratified fluid. J. Fluid Mech. 453, 371-394.

Pailha, M., Nicolas, M. \& Pouliquen, O. 2008 Initiation of underwater granular avalanches: Influence of the initial volume fraction. Phys. Fluids 20, 111701

Pailha, M. \& Pouliquen, O. 2009 A two-phase flow description of the initiation of underwater granular avalanches. J. Fluid Mechanics 633, 115-135.

Pegler, S. S. \& Worster, M. G. 2013 An experimental and theoretical study of the dynamics of grounding lines. J. Fluid Mech. 728, 5-28.

Pouliquen, O. \& Hutter, K. 2002 Friction law for dense granular flows: application to the motion of a mass down a rough inclined plane. J. Fluid Mech. 453, 133-151.

Rondon, L., Pouliquen, O. \& Aussillous, P. 2011 Granular collapse in a fluid: Role of the initial volume fraction. Phys. Fluids 23, 073301.

Savage, S. B. \& Hutter, K. 1989 The motion of a finite mass of granular material down a rough incline. J. Fluid Mech. 199, 177-215.

Simpson, J. E. 1982 Gravity currents in the laboratory, atmosphere, and ocean. Ann. Rev. Fluid Mech. 14, 213-234.

Staron, L. \& Hinch, E. J. 2005 Discrete simulation of the collapse of granular columns. $J$. Fluid Mech. 545, 1-27.

StARON, L. \& Hinch, E. J. 2007 The spreading of a granular mass: role of grain properties and initial conditions. Granular Matter 9, 205-217.

Thompson, E. L. \& Huppert, H. E. 2007 Granular column collapses: further experimental results. J. Fluid Mechanics 575, 177-186.

Topin, V., Monerie, Y., Perales, F. \& Radjai, F. 2012 Collapse dynamics and runout of dense granular materials in a fluid. Phys. Rev. Lett. 109, 188001.

Viroulet, S., Sauret, A. \& Kimmoun, O. 2014 Tsunami generated by a granular collapse down a rough inclined plane. Europhys. Lett. 105, 34004.

Zitti, G., Ancey, C., Postacchini, M. \& Brocchini, M. 2016 Impulse waves generated by snow avalances: Momentum and energy transfer to a water body. J. Geophys. Res. Earth Surf. 121, 2399-2423. 